\title{
PHARMACEUTICAL POTENTIAL OF LABORATORY GROWN CULTURES OF BLUE-GREEN ALGAE: A COMPREHENSIVE REVIEW AND FUTURE POSSIBILITIES
}

\author{
Ritu Chauhan ${ }^{1}$, Abhishek Chauhan ${ }^{2 *}$, Ashutosh Tripathi ${ }^{1^{*}}$, Anuj Ranjan ${ }^{2}$, \\ Subhash C. Chauhan ${ }^{3}$, Tanu Jindal ${ }^{1,2}$

\footnotetext{
${ }^{1}$ Amity Institute of Environmental Sciences, Amity University, Sector-125, Noida, Uttar Pradesh, India

${ }^{2}$ Amity Institute of Environmental Toxicology, Safety and Management, Amity University, Sector-125, Noida, Uttar Pradesh, India

${ }^{3}$ STCECR, School of Medicine, University of Texas Rio Grande Valley, McAllen, TX 78504, USA
}

Received - July 26, 2021; Revision - September 29, 2021; Accepted - October 16, 2021

Available Online - October 30, 2021

DOI: http://dx.doi.org/10.18006/2021.9(5).543.571

KEYWORDS
Blue-Green Algae
Biomass
Pharmaceutically Important
Compound
Biological activities
Discovery
Development

\begin{abstract}
COVID-19 pandemic has taught the world researchers the urgent need for new sources and novel pharmaceuticals not only for existing diseases but also for both seasonal epidemics and future pandemics. Pharmaceutical drug discoveries for the past fifty years depended deeply on the procedure of empirical transmission of a huge number of pure bioactive compounds to provide new leads. The screening of extracts or isolating compounds is a common way to discover novel biologically active molecules. Most of the valuable Blue-Green algal metabolites are concentrated in their biomass. For existence in nature, Blue-Green algae (BGA) secrete and contain various organic substances like proteins, fatty acids, vitamins, pigments, primary and secondary metabolites, and these compounds are explored for potential biological activities such as antibacterial, antifungal, antiviral (including the antiSARS-CoV-2 virus that causes COVID-19), anticancer, antioxidant, antidiabetic, protease inhibitory activity, anti-inflammatory activity, etc. Due to their diverse application, pharmaceutical companies have shown commercial interest in the Blue-green algal group for the discovery and development of novel molecules to combat deadly diseases for the benefit of society and mankind. The current review paper highlights and discusses the diverse pharmaceutical potential of laboratory-grown cultures of BGA along with comprehensive and current knowledge on bioactive compounds discovered by researchers globally.
\end{abstract}

* Corresponding author

E-mail: atripathi1@amity.edu (Ashutosh Tripathi); akchauhan@amity.edu (Abhishek Chauhan)

Peer review under responsibility of Journal of Experimental Biology and Agricultural Sciences.

Production and Hosting by Horizon Publisher India [HPI] (http://www.horizonpublisherindia.in/).

All rights reserved.
All the articles published by Journal of Experimental Biology and Agricultural Sciences are licensed under a Creative Commons Attribution-NonCommercial 4.0 International License Based on a work at www.jebas.org.

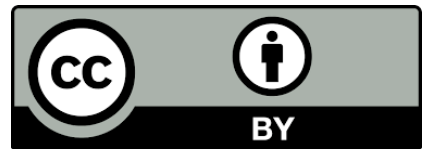




\section{Introduction}

In the extremely competitive environment of current pharmaceutical research and development of new molecules, natural products offer a unique element of molecular diversity and biological functionality, which is essential for drug discovery (Bernardini et al., 2018; Chatterjee et al., 2019; Atanasov et al., 2021). The study of secondary metabolites that organisms such as microbes including BGA and plants have evolved, largely for their survival, has historically proved of immense benefit in drug discovery and development (Petersen et al., 2020). They are providing a rich source of structurally novel bioactive molecules such as lipopeptides, amino acids, fatty acids, etc., many of which have become life-saving drugs (Singh et al., 2021a). In recent decades, pharmaceutical inventions are focused on natural sources (microbial sources such as bacterial, fungi, algal including Bluegreen algae) which can deal with recent diseases. Medicinal chemistry is the backbone of lead generation in early drug invention where small molecule hits from high throughput screen (HTS), which leads to limited optimization and identification of lead compounds (Jimenez-Lopez et al., 2021). Despite these efforts, some new chemical entities have reached the market, and researchers throughout the world now are giving more attention to exploring these groups of microorganisms (including microbes from extreme environments i.e., Arctic, Antarctic) for extractions of novel compounds with diverse pharmaceutical applications.

\subsection{The need for novel Pharmaceutically important compounds}

The less accessibility and high cost of new generation antibiotics necessitate looking for the substances from alternative medicines with claimed antimicrobial activity. Today, most of the diseases caused by pathogens can be cured with the help of available antibiotics, but the discovery of any new antibiotic generally follows up with a course of resistance mechanism building up against it among the target organisms (Dimri et al., 2018). This phenomenon is known as 'antibiotic resistance is developing among microbial species at an appreciable rate, is a formidable complication of prudent and overuse of available antibiotics, and is imposing a serious health threat to human welfare. World Health Organization (WHO) in February 2017 published a report of antimicrobial-resistant bacteria for which new pharmaceutical compounds are urgently needed (WHO, 2017). Keeping in view the urgent requirement, the current review highlights various important aspects about the pharmaceutical potential of laboratorygrown cultures of BGA.

\subsection{Blue-Green Algae}

Blue-green algae are a group of extraordinary, diverse, gramnegative, oxygenic, photosynthetic prokaryotic, microscopic oldest organisms that originated 3.5 billion years ago (Kaushik et al.,
2009). Blue-green algae are found all over the world, shows remarkable ecological diversity of habitats such as Freshwater (Khatoon et al., 2018; Chittapun et al., 2020), Terrestrial (Radzi et al., 2019; Riba et al., 2020), Marine (Basu et al., 2019; Uma et al., 2020), Hot spring (Tang et al., 2018; Cheng et al., 2020), etc. These BGA are also widely distributed in the polar region such as the Arctic, Antarctic, Southern Ocean, and Himalayas (Singh \& Elster, 2007; Rego et al., 2019; Zaki et al., 2020). It has been estimated that about 2000 strains of freshwater and marine BGA are distributed all over the world. The capability to grow in adverse conditions and their autotrophic nature makes them an eligible candidate to grow in low nutrient-deficient lakes, ponds, and oceans which pose a serious threat to water and result in eutrophication. This may cause unpleasant tastes and odors of water through the secretion of volatile compounds. Random screening of blue-green algae will continue to play an important role in the drug discovery process for the foreseeable future. Several studies have been conducted for the isolation and identification of Blue-Green Algae from water, soil, sediments, algal mats, etc. (Figure 1-8) using advanced morphological, physiological, and molecular characterization techniques (Bellinger \& Sigee, 2015; Hokmollahi et al., 2016; Radkova et al., 2020). These BGA have successfully grown on a laboratory scale using selective media i.e. BG-11, BG-13, Chu 10 (Chu, 1942; Rippka et al., 1979; Kaushik et al., 2010), Allen and Arnon Medium (Allen \& Arnon, 1955), Fogg's Medium (Fogg's. 1965), Modified Bristol's Medium (Bold, 1949) and Pringsheim's Medium (Pringsheim, 1946). Blue-green algae do not require carbon or energy sources in their growth medium. Thus, they require only a basic inorganic medium, which has several logical advantages when performing the mass culture and purification of active compounds. Flask cultivation and mass cultivation for instance open pond system, hybrid system, closed photobioreactors are very well-known culturing methods used for generating biomass maintaining proper light, temperature, water, $\mathrm{CO}_{2}$ supply, $\mathrm{pH}$, nutrient supply, and proper mixing (Kaiwan-arporn et al., 2012; Troschl et al., 2017; Al-Saman et al., 2020; Jo et al., 2020). Lyophilization (freeze-drying), air drying, and sun-drying are some known popular techniques to convert biomass into powder (Smetana et al., 2017). Aqueous extraction i.e. cold and hot water extraction, organic extraction i.e. polar solvent extraction, semi-polar solvent extraction, non-polar solvent extraction, mix solvents extraction and sequential extractions, soxhlet extraction have been used extensively to isolate medicinal value active ingredients (Fatima et al., 2017; Vanlalveni et al., 2018; Yücer et al., 2018; Saurav et al., 2019). Generally, all blue-green algae vegetative cells contain carboxysomes, pseudocrystalline aggregates of the key enzymes of $\mathrm{CO}_{2}$ fixation via the reductive pentose phosphate pathway and glycogen is a general carbohydrate reserve material of cyanobacteria. Other cellular inclusions include Poly- $\beta$ hydroxybutyrate (PHB) granules, cyanophycean granules, polyphosphate granules, carboxysomes or polyhedral bodies, and gas 
vesicles (Stanier, 1988). They show notable ecological diversity. Because of extensive eutrophication of lakes, ponds and some parts of oceans BGA often forms blooms, which lead to water hygienic problems (Chorus et al., 2000; Duy et al., 2000). They may cause unpleasant tastes and odors through the excretion of volatile compounds (Jones \& Korth, 1995; Liu et al., 2006).
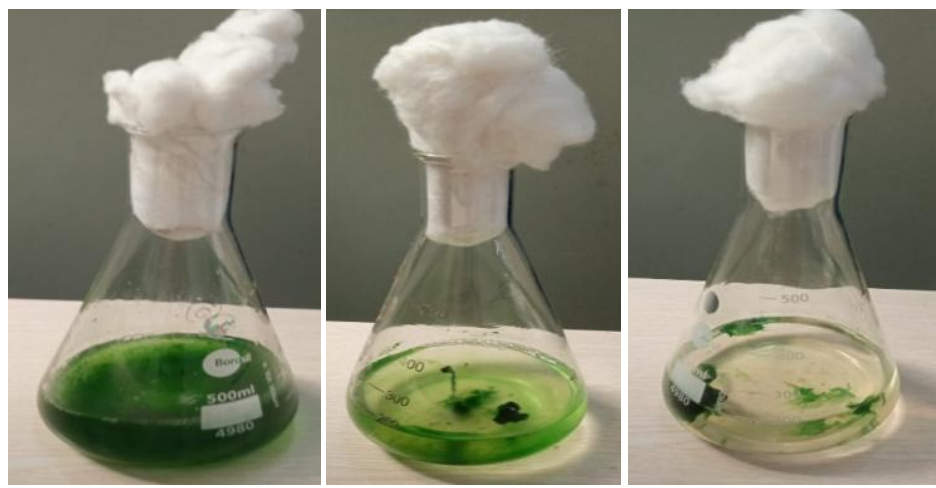

Figure 1 Laboratory Grown Culture of BGA (Medium: BG-11): A. Calothrix sp., B. Spirulina sp. C. Oscillatoria sp.
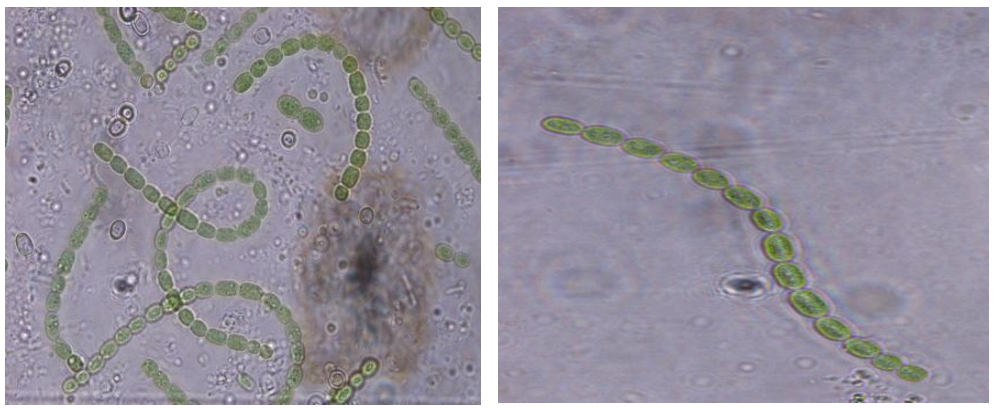

Figure 2 Microscopic images of Anabaena species (Chauhan \& Jindal, 2020)
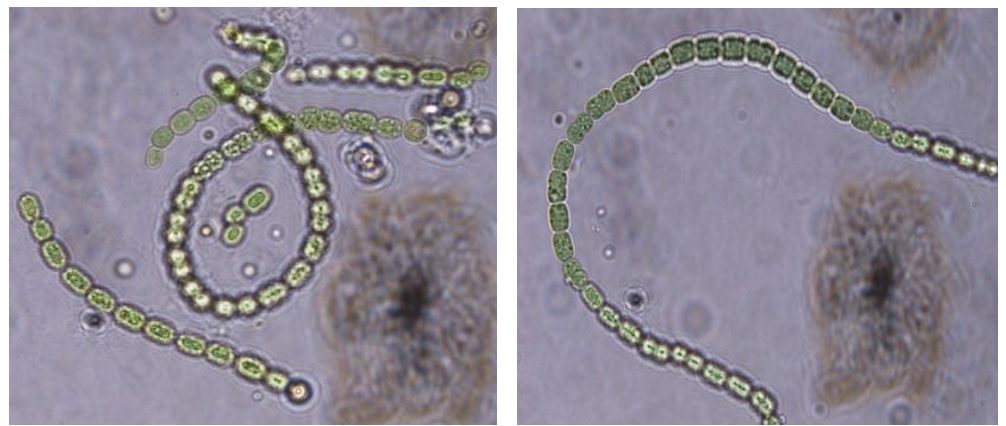

Figure 3 Microscopic images of Nostoc species (Chauhan \& Jindal, 2020)
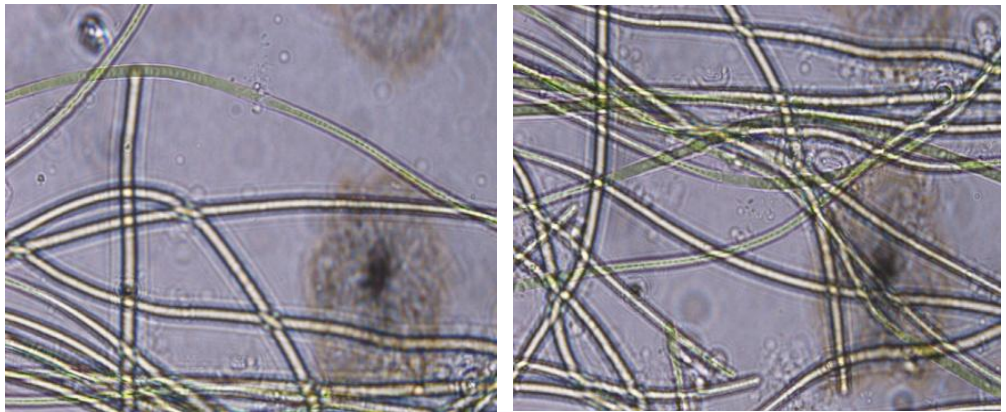

Figure 4 Microscopic images of Calothrix species (Chauhan \& Jindal, 2020)

Journal of Experimental Biology and Agricultural Sciences http://www.jebas.org 

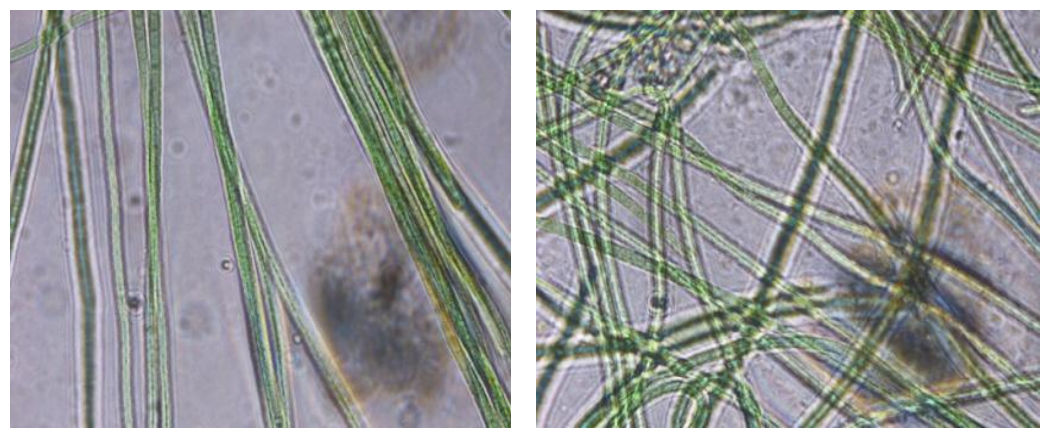

Figure 5 Microscopic images of Oscillatoria species (Chauhan \& Jindal, 2020)
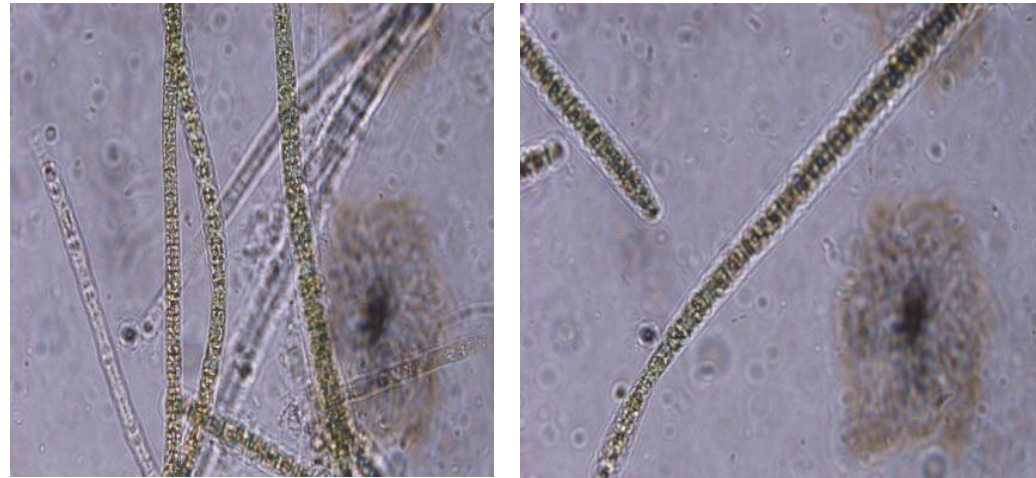

Figure 6 Microscopic images of spirulina species (Chauhan \& Jindal, 2020)
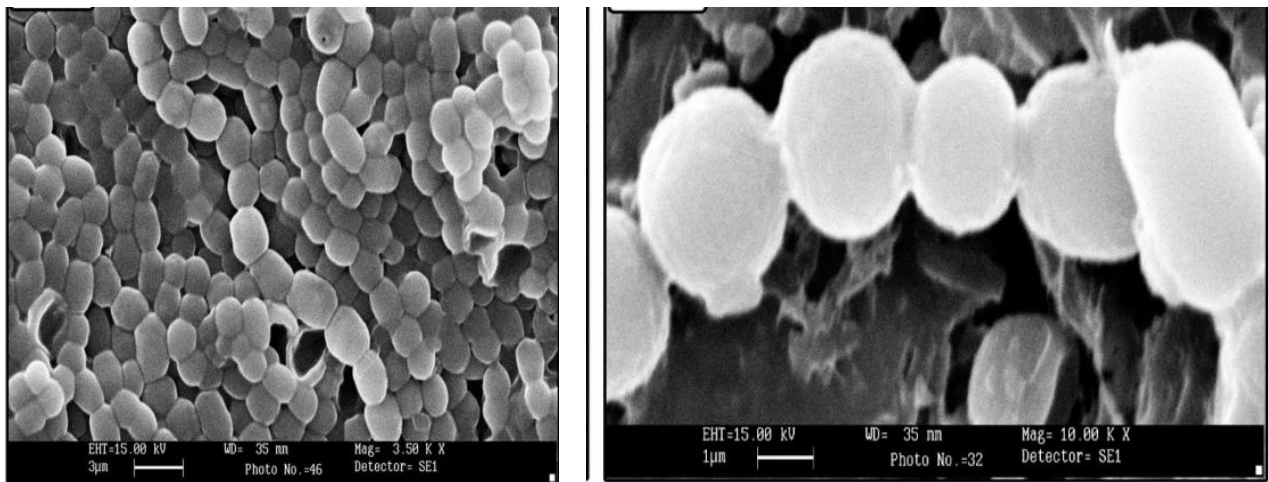

Figure 7 Scanning Electron Micrograph (SEM) image of Anabaena sp. (A) and Nostoc sp. (B) as per Kaushik \& Chauhan (2008a)

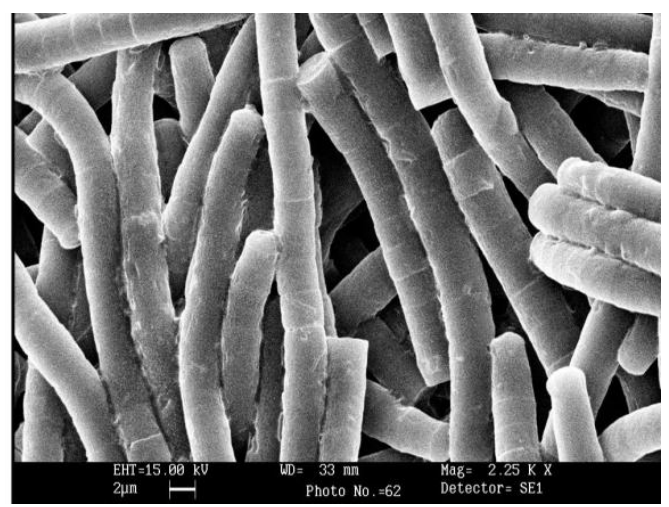

Figure 8 Scanning Electron Micrograph (SEM) image of Spirulina platensis (Kaushik \& Chauhan, 2008b)

Journal of Experimental Biology and Agricultural Sciences http://www.jebas.org 


\section{Pharmaceutical Potential of BGA}

Blue-green algae are the rich source of structurally novel and biologically active metabolites with diverse antibacterial, antifungal, antiviral (including the anti-SARS-CoV-2 virus that causes COVID-19), anticancer, antioxidant, antidiabetic, protease inhibitory activity, anti-inflammatory activity, immunomodulatory activity, larvicide, and protease inhibitory activity, etc. (Figure 9) (Nainangu et al., 2020; Jafari et al., 2021). The first time before $1500 \mathrm{BC}$, medicinal and nutritional properties have been investigated for Nostoc algal species to treat gout, fistula, and cancer (Cardellina et al., 1979a; Shishido et al., 2020).

These photosynthetic microorganisms can yield proteins, carbohydrates, and lipids as a result of photosynthesis thus referred to as important biological resources having a wide range of biotechnological applications in the modern world due to their ability to grow rapidly even in harsh environmental conditions (Padmini et al., 2021). A search of these organisms for medicinal purposes has revealed important chemical prototypes for the finding of new agents, stimulating the use of refined physical techniques and new syntheses of molecules with the pharmaceutical application for human welfare. Phytochemical's constituents described from extracts of Blue-Green algae have been described by researchers (Figure 10) and are very well documented (Vasudevan et al., 2020; Nainangu et al., 2020; Gabr et al., 2020; Vasudevan et al., 2020)

\subsection{Pharmaceutically important compounds isolated from BGA}

Secondary metabolites refer to those compounds that are not used by the organisms for their primary metabolisms. Secondary metabolites influence other organisms in the vicinity and are thought to be of phylogenetic importance (Carpine \& Sieber, 2021). Secondary metabolites include several types of compounds that may act as hormones, antibiotics, allelochemicals, toxins, and biotoxins that are found in surface supplies of fresh water (Carmichael, 1992). The ability of such compounds to kill bacteria and fungi have been well documented (Bonjouklian et al., 1988). The properties of secondary metabolites in nature are not completely understood (Metting \& Pyne, 1986; Inderjit \& Dakshini, 1994; Vasudevan et al., 2020).

The blue-green algae bear the characteristics to secrete vitamins, amino acids, fatty acids, carbohydrates, and various primary and secondary metabolites like amines, histamines, histidine, tannins, terpenoids, bromophenol, and polysaccharides (Figure 11). Few of these compounds are proven to be biologically active (Metting \& Pyne, 1986; Padmini et al., 2021). The recent examples are cyanovirin-N secreted by Nostoc ellipososoporum and anti-HIV glycolipids secreted by Isochyrosis and bromophenol are secreted by Calothrix sp. (Jaspars \& Lawton 1998; Safari et al., 2020).

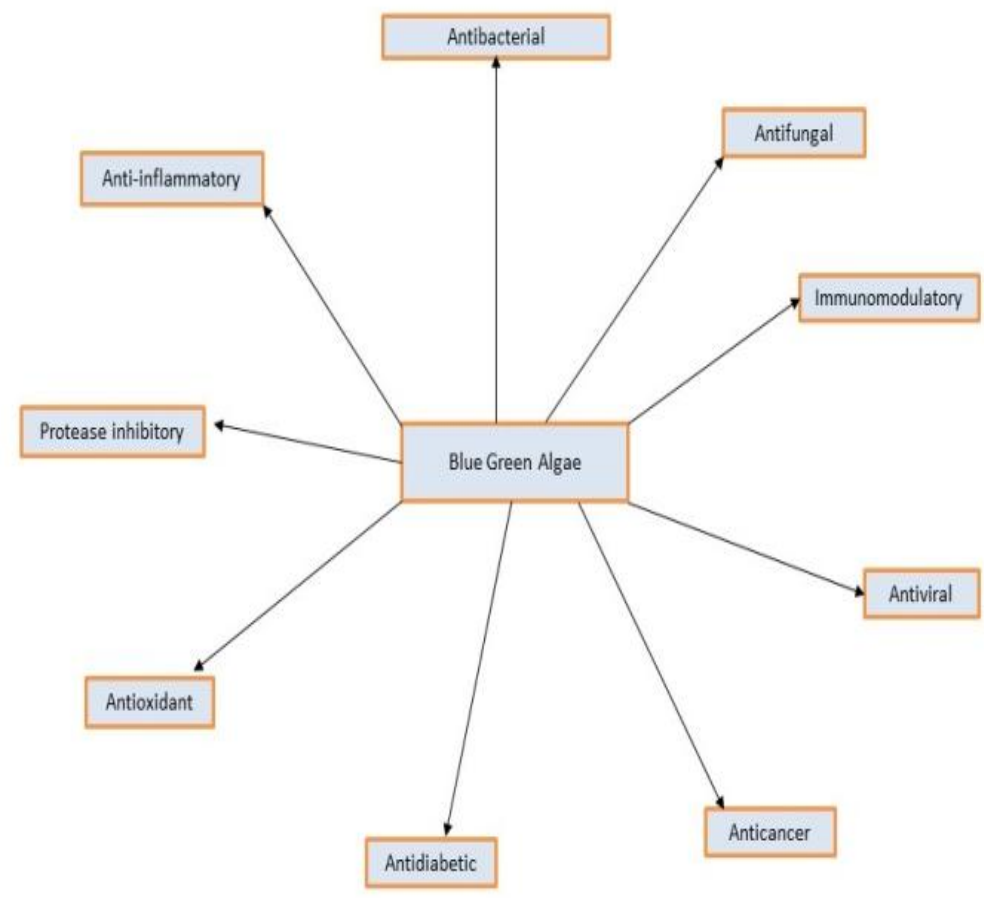

Figure 9 Pharmaceutical Potential of Blue-Green Algae

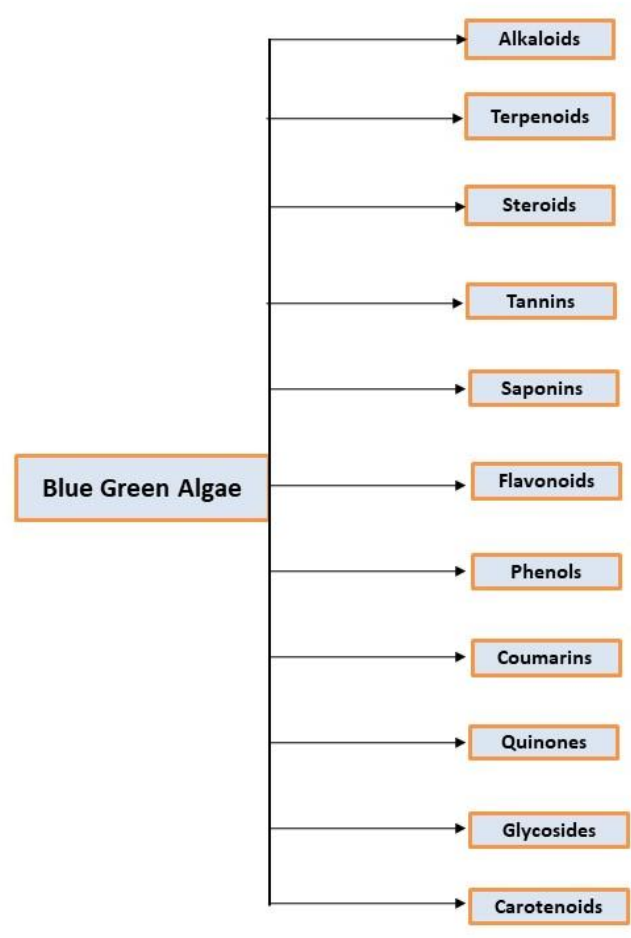

Figure 10 Major Phytochemicals constituents described from extracts of Blue-Green algae

Journal of Experimental Biology and Agricultural Sciences http://www.jebas.org 


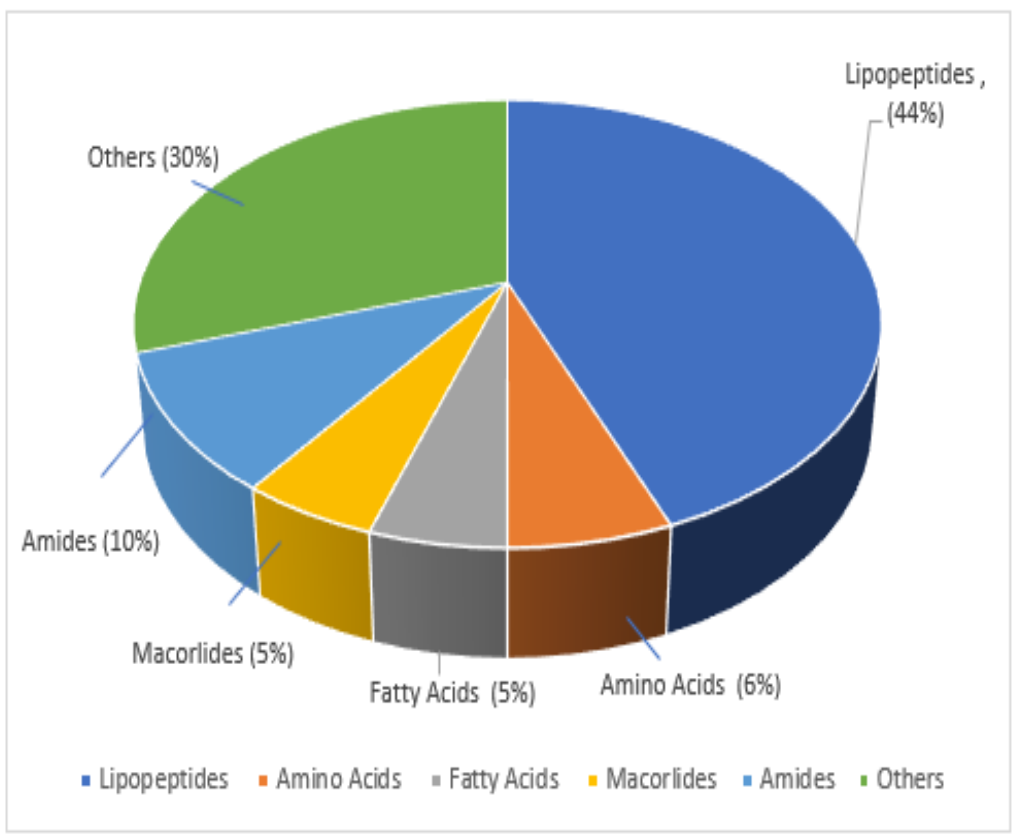

Figure 11 Pharmaceutically Important Compounds isolated from BGA

\subsubsection{Antibacterial Potential of BGA}

BGA are known for the secretions of antibacterial compounds with potential antibacterial activity against both Gram-positive and Gramnegative bacteria. Several strains such as Anabaena, Lyngbya, Calothrix, Spirulina, Nostoc, Hapalosiphone, Phormidium, and Oscillatoria have been identified by researchers from different habitats which can produce a wide variety of antibacterial molecules having therapeutic potentials (Chauhan et al., 2022). These organisms are even being altered genetically using biotechnological interventions for the production of various active compounds having antibacterial activity such as Bacteriocin Ambigol A, Parsiguine, Hapalindole, Hormothamnin A. Bacillus subtilis, Staphylococcus aureus, Streptococcus sanguinis, Pseudomonas aeruginosa, Escherichia coli, L. monocytogenes, Salmonella typhimurium, Enterobacter aerogenes, Klebsiella pneumonia, Methicillin-resistant B. anthracis are the examples of some gram-positive and Gramnegative bacteria which have studied for the inhibitory action of BGA (Luesch et al., 2001; Muller et al., 2006; Mo et al., 2009; Sturdy et al., 2010). The first partly identified antimicrobial compound isolated from algae were obtained from unicellular green algae particularly, Chlorella which contained a substance termed as 'chlorellin' that exhibited inhibitory activity against both Grampositive and Gram-negative bacteria, including Staphylococcus aureus, Streptococcus pyogenes, Bacillus subtilis, and Pseudomonas aeruginosa (Pratt et al., 1944). Chlorellin is composed of peroxides of unsaturated fatty acids (Spoehr \& Milner, 1949). Kaushik \&
Chauhan (2008a) had reported the antibacterial activity of several species of cyanobacteria such as Anabaena, Lyngbya, Calothrix, Spirulina, Nostoc, Hapalosiphone, Phormidium, and Oscillatoria, etc. against both Gram-positive (Staphylococcus aureus, Bacillus subtilis, Bacillus cereus, etc.) and Gram-negative bacteria (Escherichia coli, Pseudomonas aeruginosa, Salmonella typhi, etc.). In a similar study, various species of Anabaena were evaluated for their antimicrobial activity and active antibacterial extracts were further screened for the presence of various chemical constituents through HPTLC techniques (Kaushik et al., 2009; Chauhan et al., 2010). Extracts of Nostoc commune and Lyngbya majuscula were studied for potent antimicrobial activity against clinically significant microorganisms (Kaushik \& Chauhan, 2008a; Kaushik \& Chauhan 2008b; Kaushik et al., 2009; Verma et al., 2016). HPTLC analysis were also performed to identify novel pharmaceutical compounds responsible for the activity. In a recent study, El-Sheekh et al. (2021) have evaluated the antibacterial activity of Oscillatoria sp. and Spirulina mediated silver and gold nanoparticles. Two new antibacterial molecules namely Arachidonoyl dopamine and fluocinolone recently discovered from methanolic extracts of Arthrospira platensis, a BGA isolated from a hypersaline lake in Rajasthan, India (Singh et al., 2021b). In another study, Antibacterial efficacy extracts of Oxynema thaianum have been assessed against multi-drug-resistant bacteria such as E. coli and K. pneumoniae (Padmini et al., 2021). Antibacterial compounds discovered from various species of BGA have been listed in Table 1 and Figure 12. 
Table 1 Antibacterial compounds reported from BGA

\begin{tabular}{|c|c|c|c|}
\hline BGA Sps. & $\begin{array}{l}\text { Antibacterial } \\
\text { Compounds }\end{array}$ & Detail of Chemical compounds & References \\
\hline $\begin{array}{l}\text { Scytonema } \\
\text { pseudo } \\
\text { hofmanni }\end{array}$ & Scytophycins C & $\begin{array}{c}\text { MF: } \mathrm{C}_{45} \mathrm{H}_{75} \mathrm{NO}_{11} \\
\text { MW: } 806.1 \mathrm{~g} / \mathrm{mol} \\
\text { IUPAC Name: N-[(E,3R,4R,5R,9S,10S,11S)-10-hydroxy-11- } \\
\text { [(1S,3S,4R,5S,7R,8S,9R,12E,14E,17S,19R)-17-hydroxy-3,5,7-trimethoxy- } \\
\text { 4,8,14-trimethyl-11-oxo-10,23-dioxabicyclo[17.3.1]tricosa-12,14,20-trien- } \\
\text { 9-yl]-4-methoxy-3,5,9-trimethyl-6-oxododec-1-enyl]-N-methylformamide }\end{array}$ & $\begin{array}{c}\text { Ishibashi et al., } \\
1986\end{array}$ \\
\hline $\begin{array}{l}\text { Scytonema } \\
\text { ocellatum, } \\
\text { Tolypothrix } \\
\text { conglutinate }\end{array}$ & Tolytoxin & $\begin{array}{c}\text { MF: } \mathrm{C}_{46} \mathrm{H}_{75} \mathrm{NO}_{13} \\
\text { MW: } 850.1 \mathrm{~g} / \mathrm{mol} \\
\text { IUPAC Name: N-[(E,3R,4R,5R,9S,10S,11S)-10-hydroxy-11- } \\
\text { [(1S,3S,4S,5S,7R,8S,9R,12E,14E,16S,17R,19R)-16-hydroxy-3,5,7,17- } \\
\text { tetramethoxy-8,14-dimethyl-11-oxospiro[10,23- } \\
\text { dioxabicyclo[17.3.1]tricosa-12,14,20-triene-4,2'-oxirane]-9-yl]-4-methoxy- } \\
\text { 3,5,9-trimethyl-6-oxododec-1-enyl]-N-methylformamide }\end{array}$ & Moore, 1982 \\
\hline $\begin{array}{l}\text { Tolypothrix } \\
\text { nodosa }\end{array}$ & Tolyporphin J & $\begin{array}{c}\text { MF: } \mathrm{C}_{24} \mathrm{H}_{22} \mathrm{~N}_{4} \mathrm{O}_{4} \\
\text { MW: } 430.5 \mathrm{~g} / \mathrm{mol} \\
\text { IUPAC Name: } 3,13 \text {-dihydroxy-3,7,13,18-tetramethyl-22,24- } \\
\text { dihydroporphyrin-2,12-dione }\end{array}$ & $\begin{array}{c}\text { Prinsep et al., } \\
1992\end{array}$ \\
\hline $\begin{array}{c}\text { Fischerella } \\
\text { ambigua }\end{array}$ & Ambigol A & $\begin{array}{c}\text { MF: } \mathrm{C}_{18} \mathrm{H}_{8} \mathrm{Cl}_{6} \mathrm{O}_{3} \\
\text { MW: } 485 \mathrm{~g} / \mathrm{mol} \\
\text { IUPAC Name: 3,5-dichloro-2(3,5-dichloro-2-hydroxyphenyl)-6-(2,4- } \\
\text { dichlorophenoxy)phenol }\end{array}$ & Falch et al., 1995 \\
\hline $\begin{array}{c}\text { Nostoc } \\
\text { muscorum }\end{array}$ & Muscoride A & $\begin{array}{c}\text { MF: } \mathrm{C}_{28} \mathrm{H}_{40} \mathrm{~N}_{4} \mathrm{O}_{5} \\
\text { MW: } 512.6 \mathrm{~g} / \mathrm{mol} \\
\text { IUPAC Name: 3-methylbut-2-enyl 5-methyl-2-[5-methyl-2-[1-[3-methyl- } \\
\text { 2-(2-methylbut-3-en-2-ylamino)butanoyl]pyrrolidin-2-yl]-1,3-oxazol-4- } \\
\text { yl]-1,3-oxazole-4-carboxylate }\end{array}$ & $\begin{array}{c}\text { Nagatsu et al., } \\
1995\end{array}$ \\
\hline $\begin{array}{c}\text { Fischerella } \\
\text { ambigua }\end{array}$ & Tjipanazole D & $\begin{array}{c}\text { Compound CID: } 10087661 \\
\text { MF: } \mathrm{C}_{18} \mathrm{H}_{10} \mathrm{Cl}_{2} \mathrm{~N}_{2} \\
\text { MW: } 325.2 \mathrm{~g} / \mathrm{mol} \\
\text { IUPAC Name: 3,8-dichloro-11,12-dihydroindolo[2,3-a] carbazole }\end{array}$ & Falch et al., 1995 \\
\hline $\begin{array}{l}\text { Microcystis } \\
\text { aeruginosa }\end{array}$ & Kawaguchipeptin A & $\begin{array}{c}\text { MF: } \mathrm{C}_{68} \mathrm{H}_{92} \mathrm{~N}_{16} \mathrm{O}_{18} \\
\text { MW: } 1421.6 \mathrm{~g} / \mathrm{mol} \\
\text { IUPAC Name: 2- } \\
\text { [(3S,9S,12S,15S,17S,25R,28S,31S,34S,40S,43R,46S,48S,56S)-28,31,40- } \\
\text { tris(2-amino-2-oxoethyl)-9-[(1R)-1-hydroxyethyl]-12-(hydroxymethyl)- } \\
\text { 17,48-bis(3-methylbut-2-enyl)-43-(2-methylpropyl)- } \\
2,8,11,14,27,30,33,36,39,42,45 \text {-undecaoxo- } \\
1,7,10,13,24,26,29,32,35,38,41,44,55- \\
\text { tridecazaoctacyclo[44.10.0.03,7.015,26.017,25.018,23.048,56.049,54]hexa } \\
\text { pentaconta-18,20,22,49,51,53-hexaen-34-yl]acetic acid }\end{array}$ & Ishida et al., 1997 \\
\hline $\begin{array}{l}\text { Nostoc } \\
\text { spongiaeforme } \\
\text { var. tenue }\end{array}$ & Tenuecyclamide A & $\begin{array}{c}\text { MF: } \mathrm{C}_{19} \mathrm{H}_{20} \mathrm{~N}_{6} \mathrm{O}_{4} \mathrm{~S}_{2} \\
\text { MW: 460.5g/mol } \\
\text { IUPAC Name: (4S)-4,7,11,18-tetramethyl-6-oxa-13,20-dithia- } \\
3,10,17,22,23,24 \text {-hexazatetracyclo[17.2.1.15,8.112,15]tetracosa- } \\
\text { 1(21),5(24),7,12(23),14,19(22)-hexaene-2,9,16-trione } \\
\end{array}$ & $\begin{array}{c}\text { Banker \& } \\
\text { Carmeli, } 1998\end{array}$ \\
\hline $\begin{array}{l}\text { Nostoc } \\
\text { commune }\end{array}$ & $\begin{array}{l}\text { 1,8-dihydroxy-4- } \\
\text { methyl } \\
\text { anthraquinone } \\
\end{array}$ & $\begin{array}{c}\text { MF: } \mathrm{C}_{15} \mathrm{H}_{10} \mathrm{O}_{4} \\
\text { MW: } 254.24 \mathrm{~g} / \mathrm{mol} \\
\text { IUPAC Name: 4,5-dihydroxy-1-methylanthracene-9,10-dione }\end{array}$ & Jaki et al., 2000 \\
\hline Calothrix sp. & Calothrixin A & $\begin{array}{c}\text { MF: } \mathrm{C}_{19} \mathrm{H}_{10} \mathrm{~N}_{2} \mathrm{O}_{3} \\
\text { MW: } 314.3 \mathrm{~g} / \mathrm{mol} \\
\text { IUPAC Name: } 20 \text {-oxido-10-aza-20- } \\
\text { azoniapentacyclo[11.8.0.03,11.04,9.014,19]henicosa- } \\
\text { 1(13),3(11),4,6,8,14,16,18,20-nonaene-2,12-dione }\end{array}$ & Doan et al.,2000 \\
\hline
\end{tabular}

Journal of Experimental Biology and Agricultural Sciences http://www.jebas.org 


\begin{tabular}{|c|c|c|c|}
\hline BGA Sps. & $\begin{array}{l}\text { Antibacterial } \\
\text { Compounds }\end{array}$ & Detail of Chemical compounds & References \\
\hline $\begin{array}{c}\text { Nostoc } \\
\text { commune }\end{array}$ & Comnastin A & $\begin{array}{c}\text { MF: } \mathrm{C}_{27} \mathrm{H}_{40} \mathrm{O}_{4} \\
\text { MW: 428.6g/mol } \\
\text { IUPAC Name: 3-[[(3R,3aR,5aS,6R,7R,9aS,9bR)-3-(hydroxymethyl)- } \\
\text { 3,3a,6,7,9a-pentamethyl-2,4,5,5a,7,8,9,9b-octahydro-1H- } \\
\text { cyclopenta[a]naphthalen-6-yl]methyl]-4-hydroxybenzoic acid } \\
\end{array}$ & $\begin{array}{c}\text { Jaki et al., } \\
\text { Jaki et al., } 2000\end{array}$ \\
\hline Nostoc sp. & Nostocyclyne A & $\begin{array}{c}\text { MF: } \mathrm{C}_{23} \mathrm{H}_{34} \mathrm{O}_{2} \\
\text { MW: } 342.5 \mathrm{~g} / \mathrm{mol} \\
\text { IUPAC Name: 15-propylbicyclo[14.2.2]icosa-1(19),16(20),17-trien-2-yne- } \\
\text { 17,20-diol }\end{array}$ & $\begin{array}{c}\text { Ploutno \& } \\
\text { Carmeli, } 2000\end{array}$ \\
\hline $\begin{array}{c}\text { Nostoc } \\
\text { spongiaeforme }\end{array}$ & Nostocine A & $\begin{array}{c}\text { MF: } \mathrm{C}_{5} \mathrm{H}_{5} \mathrm{~N}_{5} \mathrm{O} \\
\text { MW: } 151.13 \mathrm{~g} / \mathrm{mol} \\
\text { IUPAC Name: 7-methyl-2H-pyrazolo[4,3-e][1,2,4]triazin-3-one }\end{array}$ & Hirata et al., 2003 \\
\hline $\begin{array}{l}\text { Oscillatoria } \\
\text { redekei }\end{array}$ & $\begin{array}{l}\text { a-dimorphecolic } \\
\text { acid }\end{array}$ & $\begin{array}{c}\text { MF: } \mathrm{C}_{18} \mathrm{H}_{32} \mathrm{O}_{3} \\
\text { MW: } 296.4 \mathrm{~g} / \mathrm{mol} \\
\text { IUPAC Name: (9S,10E,12Z)-9-hydroxyoctadeca-10,12-dienoic acid }\end{array}$ & Mundt et al., 2003 \\
\hline $\begin{array}{l}\text { Scytonema } \\
\text { hofmanni PCC } \\
7110\end{array}$ & Scyptolin A & $\begin{array}{c}\text { MF: } \mathrm{C}_{45} \mathrm{H}_{69} \mathrm{ClN}_{8} \mathrm{O}_{14} \\
\text { MW: } 981.5 \mathrm{~g} / \mathrm{mol} \\
\text { IUPAC Name: (2S,3R)-2-[[(2S)-2-(butanoylamino)propanoyl]amino]-N- } \\
\text { [(2S,5S,8S,11R,12S,15S,18R,21R)-5-[(3-chloro-4-hydroxyphenyl)methyl]- } \\
\text { 21-hydroxy-2-[(1R)-1-hydroxyethyl]-4,11-dimethyl-15-(2-methylpropyl)- } \\
\text { 3,6,9,13,16,22-hexaoxo-8-propan-2-yl-10-oxa-1,4,7,14,17- } \\
\text { pentazabicyclo[16.3.1]docosan-12-yl]-3-hydroxybutanamide }\end{array}$ & $\begin{array}{c}\text { MacMillan \& } \\
\text { Molinski, 2005; } \\
\text { Matern et al., } 2001\end{array}$ \\
\hline Lyngbya sp. & Pahayokolide A & $\begin{array}{c}\text { MF: } \mathrm{C}_{72} \mathrm{H}_{105} \mathrm{~N}_{13} \mathrm{O}_{20} \\
\text { MW: 1472.7g/mol } \\
\text { IUPAC Name: [1-[(6R,10R,13S,19R,22S,25E,28S,31Z,34S,37S)-6-(3-amino- } \\
\text { 3-oxopropyl)-34-benzyl-25,31-di(ethylidene)-9-hydroxy-22-[(1R)-1- } \\
\text { hydroxyethyl]-28-(hydroxymethyl)-2,5,8,12,18,21,24,27,30,33,36- } \\
\text { undecaoxo-19-(2-phenylethyl)-1,4,7,11,17,20,23,26,29,32,35- } \\
\text { undecazatricyclo[35.3.0.013,17]tetracontan-10-yl]-4,5-dihydroxy-7- } \\
\text { methyloctan-2-yl] (2S)-2-[acetyl(methyl)amino]-4-methylpentanoate }\end{array}$ & $\begin{array}{c}\text { Berry } \\
\text { Berry et al., } 2004\end{array}$ \\
\hline $\begin{array}{c}\text { Microcoleous } \\
\text { lacustris }\end{array}$ & Abietane & $\begin{array}{c}\text { MF: } \mathrm{C}_{20} \mathrm{H}_{36} \\
\text { MW: } 276.5 \mathrm{~g} / \mathrm{mol} \\
\text { IUPAC Name: }(2 \mathrm{~S}, 4 \mathrm{aS}, 4 \mathrm{bR}, 8 \mathrm{aS}, 10 \mathrm{aS})-4 \mathrm{~b}, 8,8 \text {-trimethyl-2-propan-2-yl- } \\
1,2,3,4,4 \mathrm{a}, 5,6,7,8 \mathrm{a}, 9,10,10 \mathrm{a} \text {-dodecahydrophenanthrene }\end{array}$ & $\begin{array}{l}\text { Thajuddin \& } \\
\text { Subramanian, } \\
2005\end{array}$ \\
\hline Fischerella sp. & Hapalindole T & $\begin{array}{c}\text { MF: } \mathrm{C}_{21} \mathrm{H}_{23} \mathrm{ClN}_{2} \mathrm{OS} \\
\text { MW: } 386.9 \mathrm{~g} / \mathrm{mol} \\
\text { IUPAC Name: (2S,6S,7R,8R,10R)-8-chloro-7-ethenyl-7,11,11-trimethyl-3- } \\
\text { thia-5,17-diazapentacyclo[10.6.1.02,6.02,10.016,19]nonadeca- } \\
\text { 1(18),12(19),13,15-tetraen-4-one } \\
\end{array}$ & $\begin{array}{l}\text { Asthana et al., } \\
2009\end{array}$ \\
\hline $\begin{array}{l}\text { Fischerella } \\
\text { ambigua }\end{array}$ & Ambigol B & $\begin{array}{c}\text { MF: } \mathrm{C}_{18} \mathrm{H}_{8} \mathrm{Cl}_{6} \mathrm{O}_{3} \\
\text { MW: } 485 \mathrm{~g} / \mathrm{mol} \\
\text { IUPAC Name: 3,5-dichloro-2,6-bis(2,4-dichlorophenoxy)phenol }\end{array}$ & $\begin{array}{c}\text { Raveh \& } \\
\text { Carmeli, } 2007\end{array}$ \\
\hline Nostoc sp. & $\begin{array}{c}\text { Carbamidocyclo } \\
\text { phane A }\end{array}$ & $\begin{array}{c}\text { MF: } \mathrm{C}_{38} \mathrm{H}_{54} \mathrm{Cl}_{4} \mathrm{~N}_{2} \mathrm{O}_{8} \\
\text { MW: } 808.6 \mathrm{~g} / \mathrm{mol} \\
\text { IUPAC Name: [(2R,3S,13R,14S)-13-carbamoyloxy-8,19-bis(4,4- } \\
\text { dichlorobutyl)-10,21,24,26-tetrahydroxy-3,14-dimethyl-2- } \\
\text { tricyclo[18.2.2.29,12]hexacosa-1(22),9,11,20,23,25-hexaenyl] carbamate }\end{array}$ & Bui et al., 2007 \\
\hline Nostoc sp. & $\begin{array}{l}\text { Nostocarboline } \\
\text { Hydroiodide; } \\
\text { Nostacarboline } \\
\text { Iodide }\end{array}$ & $\begin{array}{c}\text { MF: } \mathrm{C}_{12} \mathrm{H}_{10} \mathrm{CIIN}_{2} \\
\text { MW: } 344.58 \mathrm{~g} / \mathrm{mol} \\
\text { IUPAC Name: 6-chloro-2-methyl-9H-pyrido[3,4-b]indol-2-ium;iodide }\end{array}$ & $\begin{array}{c}\text { Becher et al., } \\
2007\end{array}$ \\
\hline $\begin{array}{c}\text { Fischerella } \\
\text { ambigua }\end{array}$ & $\begin{array}{c}\text { Ambiguine A } \\
\text { isonitrile }\end{array}$ & $\begin{array}{c}\text { MF: } \mathrm{C}_{26} \mathrm{H}_{31} \mathrm{ClN}_{2} \\
\text { MW: 407g/mol } \\
\text { IUPAC Name: (2S,3R,4R,5R,7S)-5-chloro-4-ethenyl-3-isocyano-4,8,8- } \\
\text { trimethyl-15-(2-methylbut-3-en-2-yl)-14- } \\
\text { azatetracyclo[7.6.1.02,7.013,16]hexadeca-1(15),9(16),10,12-tetraene }\end{array}$ & Mo et al., 2009 \\
\hline
\end{tabular}

Journal of Experimental Biology and Agricultural Sciences http://www.jebas.org 


\begin{tabular}{|c|c|c|c|}
\hline BGA Sps. & $\begin{array}{l}\text { Antibacterial } \\
\text { Compounds }\end{array}$ & Detail of Chemical compounds & References \\
\hline $\begin{array}{l}\text { Fischerella } \\
\text { ambigua }\end{array}$ & $\begin{array}{l}\text { Ambiguine A } \\
\text { isonitrile }\end{array}$ & $\begin{array}{c}\text { MF: } \mathrm{C}_{26} \mathrm{H}_{31} \mathrm{ClN}_{2} \\
\text { MW: } 407 \mathrm{~g} / \mathrm{mol} \\
\text { IUPAC Name: (2S,3R,4R,5R,7S)-5-chloro-4-ethenyl-3-isocyano-4,8,8- } \\
\text { trimethyl-15-(2-methylbut-3-en-2-yl)-14- } \\
\text { azatetracyclo[7.6.1.02,7.013,16]hexadeca-1(15),9(16),10,12-tetraene }\end{array}$ & Mo et al., 2009 \\
\hline $\begin{array}{c}\text { Leptolyngbya } \\
\text { crosbyana }\end{array}$ & Crossbyanol A & $\begin{array}{c}\text { MF: } \mathrm{C}_{30} \mathrm{H}_{15} \mathrm{Br}_{7} \mathrm{O}_{6} \\
\text { MW: } 1030.8 \mathrm{~g} / \mathrm{mol} \\
\text { IUPAC Name: 3-bromo-4-[2-bromo-4-(3-bromo-4-hydroxyphenoxy)-6-(2,4- } \\
\text { dibromophenoxy)phenoxy]-2-(2,4-dibromophenoxy)phenol }\end{array}$ & Choi et al., 2010 \\
\hline $\begin{array}{l}\text { Nostoc sp. } \\
\text { MGL001 }\end{array}$ & 9-Ethylin & $\begin{array}{l}\text { omethyl-12-(morpholin-4- ylmethoxy) 5, 8, 13, 16-tetraaza-hexacene-2, } 3 \\
\text { dicarboxy-licacid (EMTAHDCA) }\end{array}$ & $\begin{array}{l}\text { Niveshika et al., } \\
2016\end{array}$ \\
\hline
\end{tabular}

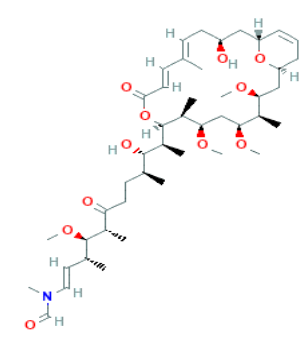

Scytophycins C

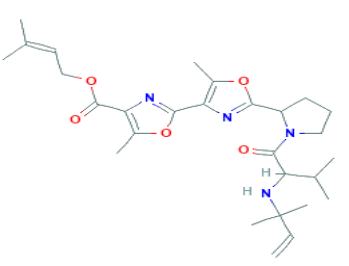

Muscoride A

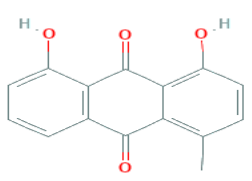

1,8-dihydroxy-4-methyl anthraquinone<smiles>Cn1ncnc2c(=O)[nH]nc1-2</smiles>

Nostocine A

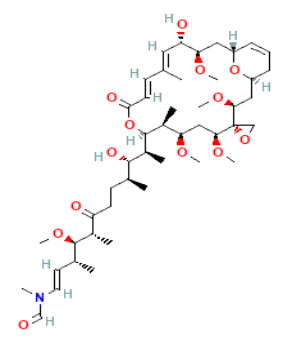

Tolytoxin

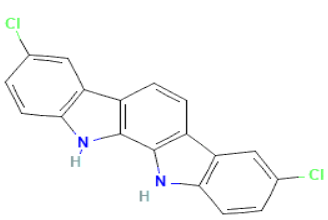

Tjipanazole D

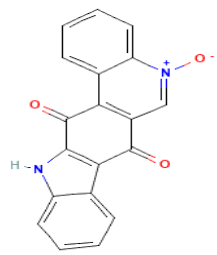

Calothrixin A

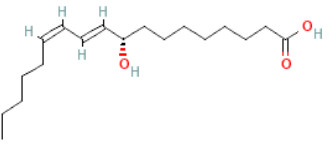

a-dimorphecolic acid

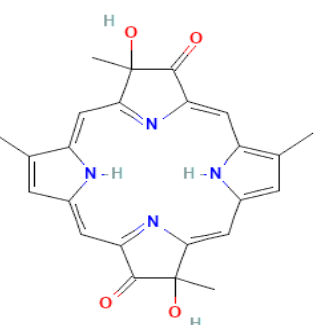

Tolyporphin J

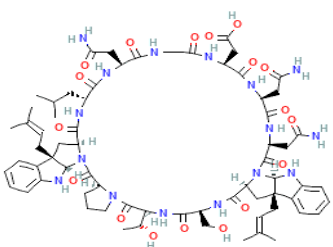

Kawaguchipeptin A

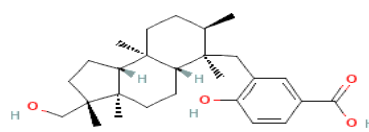

Comnastin A

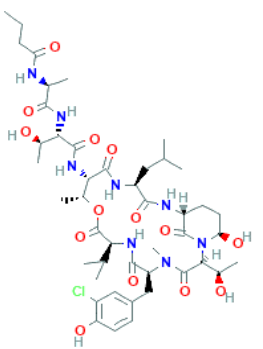

Scyptolin A<smiles>Cc1ccc(Oc2c(Cl)cc(O)c(-c3cc(Cl)cc(Cl)c3O)c2O)c(Cl)c1</smiles>

Ambigol A

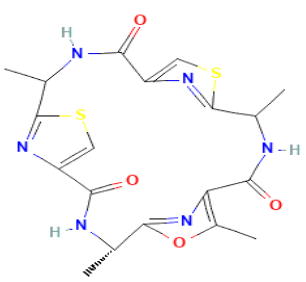

Tenuecyclamide A<smiles>CCCCCCCOc1cc(CCCCC)cc(O)c1C1CCCC1</smiles>

Nostocyclyne A

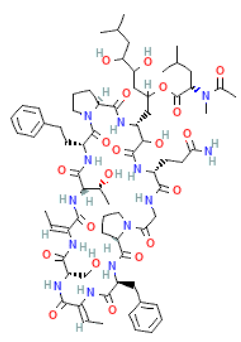

Pahayokolide A

Journal of Experimental Biology and Agricultural Sciences http://www.jebas.org 


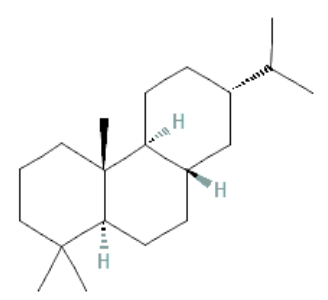

Abietane

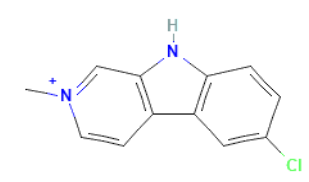

Nostocarboline Hydroiodide; Nostacarboline Iodide

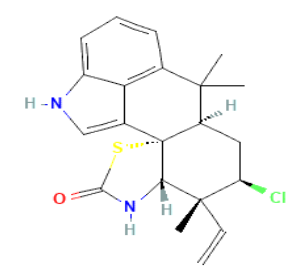

Hapalindole T

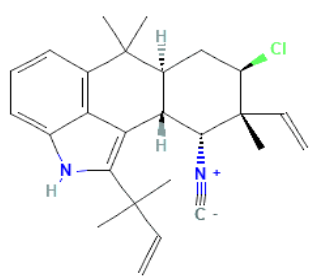

Ambiguine A isonitrile

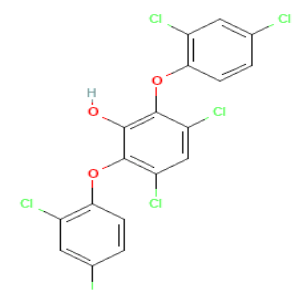

Ambigol B

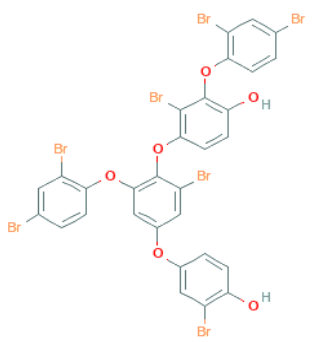

Crossbyanol A

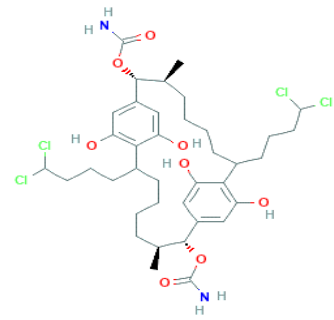

9-Ethyliminomethyl-12(morpholin-4- ylmethoxy) 5, 8, 13, 16-tetraaza-hexacene-2, 3 dicarboxy-licacid (EMTAHDCA)

Figure Not available

Figure 12 Chemical structures of the active ingredients isolated from various BGA and having antibacterial potential

\subsubsection{Antimycobacterial potential of BGA}

BGA extracts and compounds have been tested against various species of Mycobacteria. Rao et al. (2007) reported the antimycobacterial activity of different spp. of BGA viz., Hapalosiphon sp., Anabaena sp. Lyngbya sp., Westeillopsis prolifica, Spirulina sp. Anabaena variabiles, Anabaena cylindrica, Oscillatoria sp. andScytonema $s p$. against Mycobacterium tuberculosis ATCC 27294, M. tuberculosis MDR, M.avium, M. intracellulare, and M. aurum. Other BGA species which exhibit antimycobacterial potential are Tychonema sp., Fischerella ambigua are Lyngbyama juscule (Muller et al., 2006; Mo et al., 2009; Sturdy et al., 2010; Luesch et al., 2001). Antimycobacterial compounds produced by these BGA strains are summarized in Table 2 .

\subsubsection{Antifungal potential of BGA}

Antifungal properties of BGA strains have been documented globally. Fungal and yeast strains such as Candida friedricki, Fusarium oxysporum, Aspergillus fumigatus, Alternaria alternate, A. niger, C. albicans, A. parasiticus, A. flavus, A. westerdijikia, A. ochraceus ITAL 14, A. carbonarius ITAL 204, A. steynii IBT LKN 23096, Penicillium verrucosum BFE 500, $F$. verticillioides ITEM 10027, F. proliferatum MPVP 328 have been tested with BGA extracts and compounds (Marrez \& Sultan, 2016; Vanlalveni et al., 2018; Saurav et al., 2019). Antifungal compounds reported from BGA strains have been listed in Table 3 and Figure 13.

\subsubsection{Antioxidant potential of BGA}

Blue-green algae produce large amounts of antioxidants to protect themselves from harmful stress conditions so that the cells can be protected from the effect of the reactive oxygen species (ROS) produced during stress. Hydrogen peroxide and oxygen free radicals are the two harmful reactive oxygen species formed in the cells during oxidative stress and can damage the cells (Vasudevan et al., 2020). Antioxidant properties of various extracts have been evaluated using different assay methods such as DPPH radicalscavenging Assay, $\mathrm{ABTS}^{+}$(2, 2-azino-bis (3-ethylbenzthiazoline-6sulfonic acid); Nitric oxide radical scavenging Assay; Total Antioxidant capacity determination kit, and $\beta$-carotene bleaching assay (Table 5). BGA produces several antioxidants that can scavenge these free radicals. These antioxidants have been explored as a novel source of dietary supplements because the cyanobacteria are rich in phenolics, vitamins, and carotenoids, the most common being carotenoids, which can be used for alleviating oxidative stress and limiting health problems (Nainangu et al., 2020; Gabr et al., 2020). ROS are also formed in animals and humans during oxidative stress and cause damage to the biomolecules such as lipids, proteins, and DNA. Oxidative stress is associated with several diseases such as cancer, neurodegeneration, retinopathy, aging, and other diseases. Algae produce several substances that have antioxidant effects, and these substances can be used as dietary supplements by humans (Guerreiro et al., 2020; Safari et al., 2020; Li et al., 2020). Antioxidant compounds recently purified from Blue-Green Algae are summarized in Table 4. 
Table 2 Antimycobacterial compounds reported from BGA

BGA Spp. Antimycobacterial Compounds

Brunsvicamide A

MF: $\mathrm{C}_{45} \mathrm{H}_{64} \mathrm{~N}_{8} \mathrm{O}_{8}$

MW: $845 \mathrm{~g} / \mathrm{mol}$

Tychonema IUPAC Name: (2S)-2-[[(3S,6S,9S, 12S,15S)-3-benzyl-6-

sp.

(1H-indol-3-ylmethyl)-7-methyl-9-(2-methylpropyl)-

2,5,8,11,14-pentaoxo-12-propan-2-yl-1,4,7,10,13-

pentazacyclononadec-15-yl]carbamoylamino]-3-

methylpentanoic acid

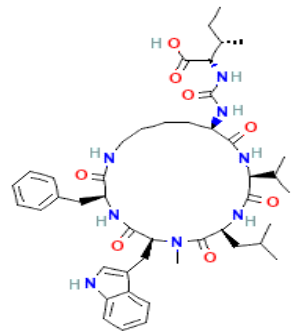

Muller et al., 2006

\section{Brunsvicamide $\mathrm{B}$ \\ MF: $\mathrm{C}_{46} \mathrm{H}_{66} \mathrm{~N}_{8} \mathrm{O}_{8}$ \\ MW: $859.1 \mathrm{~g} / \mathrm{mol}$}

Tychonema IUPAC Name: (2S)-2-[[(3S,6S,9S, 12S, 15S)-3-benzyl-

sp. 12-butan-2-yl-6-(1H-indol-3-ylmethyl)-7-methyl-9-(2methylpropyl)-2,5,8,11,14-pentaoxo-1,4,7,10,13pentazacyclononadec-15-yl]carbamoylamino]-3methylpentanoic acid

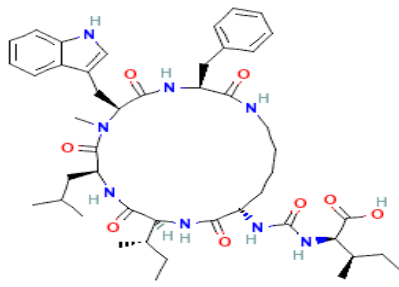

Muller et al., 2006

Eucapsitrione

MF: $\mathrm{C}_{21} \mathrm{H}_{10} \mathrm{O}_{6}$

MW: $358.3 \mathrm{~g} / \mathrm{mol}$

IUPAC Name: 2,8,19-

ambigua

trihydroxypentacyclo[11.8.0.03,11.04,9.015,20]henicosa-

1(13),2,4(9),5,7,11,15(20),16,18-nonaene-10,14,21-

trione

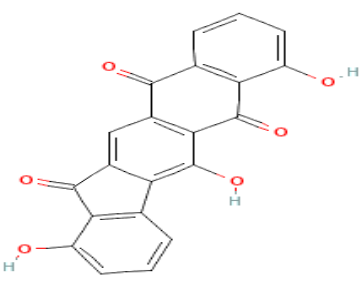

Sturdy et al., 2010

Pitipeptolide A

MF: $\mathrm{C}_{44} \mathrm{H}_{65} \mathrm{~N}_{5} \mathrm{O}_{9}$

MW: $808 \mathrm{~g} / \mathrm{mol}$

Lyngbya IUPAC Name: (3S,6S,9S,13S,19S,22S)-6-benzyl-3,19-

majuscula bis[(2S)-butan-2-yl]-7,12,12-trimethyl-13-pent-4-ynyl-9propan-2-yl-4,14-dioxa-1,7,10,17,20-

pentazabicyclo[20.3.0]pentacosane-2,5,8,11,15,18,21heptone

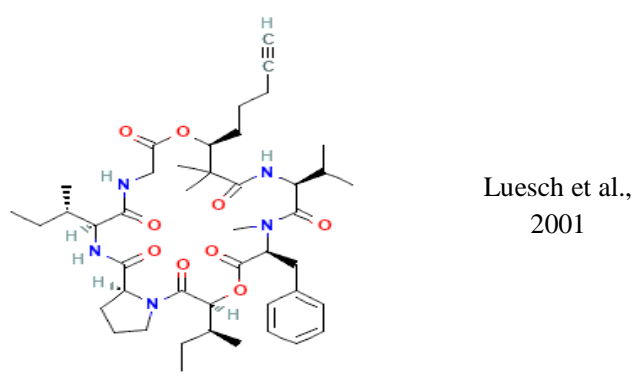

Pitipeptolides C

MF: $\mathrm{C}_{44} \mathrm{H}_{69} \mathrm{~N}_{5} \mathrm{O}_{9}$

MW: $812 \mathrm{~g} / \mathrm{mol}$

Lyngbya IUPAC Name: (3S,6S,9S,13S,19S,22S)-6-benzyl-3,19-

majuscula bis[(2S)-butan-2-yl]-7,12,12-trimethyl-13-pentyl-9-

propan-2-yl-4,14-dioxa-1,7,10,17,20-

pentazabicyclo[20.3.0]pentacosane-2,5,8,11,15,18,21-

heptone

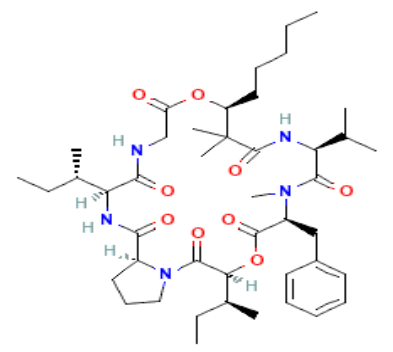

Mo et al., 2009

Journal of Experimental Biology and Agricultural Sciences

http://www.jebas.org 
Table 3 Antifungal compounds reported from BGA

\begin{tabular}{|c|c|c|c|}
\hline BGA Spp. & Antifungal Compounds & Detail of Chemical compounds & References \\
\hline Hyella caespitosa & Carazostatin & $\begin{array}{c}\text { MF: } \mathrm{C}_{20} \mathrm{H}_{25} \mathrm{NO} \\
\text { MW: } 295.4 \mathrm{~g} / \mathrm{mol} \\
\text { IUPAC Name: 1-heptyl-2-methyl-9H-carbazol-3-ol }\end{array}$ & $\begin{array}{c}\text { Cardellina et } \\
\text { al., 1979a }\end{array}$ \\
\hline $\begin{array}{l}\text { Scytonema } \\
\text { hofmanni }\end{array}$ & Cyanobacterin & $\begin{array}{c}\text { MF: } \mathrm{C}_{23} \mathrm{H}_{23} \mathrm{ClO}_{6} \\
\text { MW: } 430.9 \mathrm{~g} / \mathrm{mol} \\
\text { IUPAC Name: (5Z)-3-[(7-chloro-1,3-benzodioxol-5-yl)methyl]-4- } \\
\text { hydroxy-5-[(4-methoxyphenyl)methylidene]-4-propan-2-yloxolan-2-one }\end{array}$ & $\begin{array}{l}\text { Mason et al., } \\
1982\end{array}$ \\
\hline Tolypothrix tenuis & Toyocamycin & $\begin{array}{c}\text { MF: } \mathrm{C}_{12} \mathrm{H}_{13} \mathrm{~N}_{5} \mathrm{O}_{4} \\
\text { MW: } 291.26 \mathrm{~g} / \mathrm{mol} \\
\text { IUPAC Name: 4-amino-7-[(2R,3R,4S,5R)-3,4-dihydroxy-5- } \\
\text { (hydroxymethyl)oxolan-2-yl]pyrrolo[2,3-d]pyrimidine-5-carbonitrile }\end{array}$ & Moore, 1982 \\
\hline Tolypothrix tenuis & Tubercidin & $\begin{array}{c}\text { MF: } \mathrm{C}_{11} \mathrm{H}_{14} \mathrm{~N}_{4} \mathrm{O}_{4} \\
\text { MW: } 266.25 \mathrm{~g} / \mathrm{mol} \\
\text { IUPAC Name: }(2 \mathrm{R}, 3 \mathrm{R}, 4 \mathrm{~S}, 5 \mathrm{R})-2-(4-\text { aminopyrrolo[2,3-d]pyrimidin-7- } \\
\text { yl)-5-(hydroxymethyl)oxolane-3,4-diol }\end{array}$ & $\begin{array}{l}\text { Moore, } \\
\text { 1982; } \\
\text { Banker \& } \\
\text { Carmeli, } \\
1998\end{array}$ \\
\hline $\begin{array}{l}\text { Hapalosiphon } \\
\text { fontinalis }\end{array}$ & $\begin{array}{l}\text { Anhydrohaloxindole A; } \\
\text { Anhydrohapaloxindole A }\end{array}$ & $\begin{array}{c}\text { MF: } \mathrm{C}_{21} \mathrm{H}_{21} \mathrm{ClN}_{2} \mathrm{O} \\
\text { MW: } 352.9 \mathrm{~g} / \mathrm{mol} \\
\text { IUPAC Name: (3R,4R,5R,7R)-5-chloro-4-ethenyl-3-isocyano-4,8,8- } \\
\text { trimethyl-14-azatetracyclo[7.6.1.02,7.013,16]hexadeca-1,9(16),10,12- } \\
\text { tetraen-15-one }\end{array}$ & $\begin{array}{l}\text { Moore et al., } \\
1987\end{array}$ \\
\hline $\begin{array}{l}\text { Hapalosiphon } \\
\text { fontinalis }\end{array}$ & Fontonamide & $\begin{array}{c}\text { MF: } \mathrm{C}_{20} \mathrm{H}_{22} \mathrm{ClNO}_{2} \\
\text { MW: } 343.8 \mathrm{~g} / \mathrm{mol} \\
\text { IUPAC Name: } \mathrm{N}-[(6 \mathrm{R}, 7 \mathrm{R}, 10 \mathrm{aR})-6 \text {-chloro-7-ethenyl-7,10,10-trimethyl- } \\
\text { 9-oxo-6,10a-dihydro-5H-anthracen-1-yl]formamide }\end{array}$ & $\begin{array}{c}\text { Moore et al., } \\
1987\end{array}$ \\
\hline Nostoc sp. & Nostocyclamide & $\begin{array}{c}\text { MF: } \mathrm{C}_{20} \mathrm{H}_{22} \mathrm{~N}_{6} \mathrm{O}_{4} \mathrm{~S}_{2} \\
\text { MW: 474.6g/mol } \\
\text { IUPAC Name: (4S,18R)-4,7-dimethyl-18-propan-2-yl-6-oxa-13,20- } \\
\text { dithia-3,10,17,22,23,24-hexazatetracyclo[17.2.1.15,8.112,15]tetracosa- } \\
\text { 1(21),5(24),7,12(23),14,19(22)-hexaene-2,9,16-trione }\end{array}$ & $\begin{array}{c}\text { Moore et al., } \\
1988\end{array}$ \\
\hline $\begin{array}{l}\text { Hormothamnion } \\
\text { enteromorphoides }\end{array}$ & Hormothamnin A & $\begin{array}{c}\text { MF: } \mathrm{C}_{60} \mathrm{H}_{97} \mathrm{~N}_{11} \mathrm{O}_{14} \\
\text { MW: } 1196.5 \mathrm{~g} / \mathrm{mol} \\
\text { IUPAC Name: (3Z)-28-benzyl-19,22-di(butan-2-yl)-3-ethylidene-36- } \\
\text { hydroxy-6,31-bis(2-hydroxyethyl)-16,25-bis(2-methylpropyl)-10- } \\
\text { pentyl-1,4,7,11,14,17,20,23,26,29,32- } \\
\text { undecazabicyclo[32.3.0]heptatriacontane-2,5,8,12,15,18,21,24,27,30,33- } \\
\text { undecone }\end{array}$ & $\begin{array}{l}\text { Gerwick et } \\
\text { al., } 1989\end{array}$ \\
\hline Calothrix fusca & Calophycin & $\begin{array}{c}\text { MF: } \mathrm{C}_{19} \mathrm{H}_{18} \mathrm{~N}_{2} \mathrm{O}_{3} \\
\text { MW: } 322.4 \mathrm{~g} / \mathrm{mol} \\
\text { IUPAC Name: (3R)-2-[(4-hydroxyphenyl)methyl]-1,3,4,9- } \\
\text { tetrahydropyrido[3,4-b]indole-3-carboxylic acid }\end{array}$ & $\begin{array}{l}\text { Moon et al., } \\
1992\end{array}$ \\
\hline $\begin{array}{l}\text { Dichothrix } \\
\text { baueriana }\end{array}$ & Bauerine B & $\begin{array}{c}\text { MF: } \mathrm{C}_{12} \mathrm{H}_{8} \mathrm{Cl}_{2} \mathrm{~N}_{2} \\
\text { MW: } 251.11 \mathrm{~g} / \mathrm{mol} \\
\text { IUPAC Name: 7,8-dichloro-9-methylpyrido[3,4-b]indole }\end{array}$ & $\begin{array}{l}\text { Larsen et al., } \\
\quad 1994\end{array}$ \\
\hline $\begin{array}{c}\text { Nostoc sp. ATCC } \\
53789)\end{array}$ & Cryptophycin 1 & $\begin{array}{c}\mathrm{MF}: \mathrm{C}_{35} \mathrm{H}_{43} \mathrm{ClN}_{2} \mathrm{O}_{8} \\
\mathrm{MW}: 655.2 \mathrm{~g} / \mathrm{mol} \\
\text { IUPAC Name: (3S,6R,10R,13E,16S)-10-[(3-chloro-4- } \\
\text { methoxyphenyl)methyl]-6-methyl-3-(2-methylpropyl)-16-[(1S)-1- } \\
\text { [(2R,3R)-3-phenyloxiran-2-yl]ethyl]-1,4-dioxa-8,11-diazacyclohexadec- } \\
\text { 13-ene-2,5,9,12-tetrone }\end{array}$ & $\begin{array}{l}\text { Trimurtulu } \\
\text { et al., } 1994\end{array}$ \\
\hline $\begin{array}{l}\text { Hapalosiphon } \\
\text { welwitschii, } \\
\text { Westiella intricate }\end{array}$ & $\begin{array}{c}\text { Welwitindolinone A } \\
\text { isonitrile }\end{array}$ & $\begin{array}{c}\text { MF: } \mathrm{C}_{21} \mathrm{H}_{21} \mathrm{ClN}_{2} \mathrm{O} \\
\text { MW: } 352.9 \mathrm{~g} / \mathrm{mol} \\
\text { IUPAC Name: (3S,3'S,4'R,6'R)-4'-chloro-3'-ethenyl-2'-isocyano-3',7',7'- } \\
\text { trimethylspiro[1H-indole-3,8'-bicyclo[4.2.0]oct-1-ene]-2-one }\end{array}$ & $\begin{array}{l}\text { Stratmann et } \\
\text { al., } 1994\end{array}$ \\
\hline
\end{tabular}

Journal of Experimental Biology and Agricultural Sciences http://www.jebas.org 


\begin{tabular}{|c|c|c|c|}
\hline BGA Spp. & $\begin{array}{l}\text { Antifungal } \\
\text { Compounds }\end{array}$ & Detail of Chemical compounds & References \\
\hline $\begin{array}{l}\text { Nostoc } \\
\text { commune }\end{array}$ & Nostofungicidine & $\begin{array}{c}\text { MF: } \mathrm{C}_{48} \mathrm{H}_{76} \mathrm{~N}_{10} \mathrm{O}_{18} \\
\text { MW: } 1081.2 \mathrm{~g} / \mathrm{mol} \\
\text { IUPAC Name: 2-[16-(2-amino-1-hydroxy-2-oxoethyl)-27-hydroxy-19-[hydroxy-(4- } \\
\text { hydroxyphenyl)methyl]-3,22-bis(hydroxymethyl)-10-(3-hydroxypentadecyl)- } \\
\text { 2,5,8,12,15,18,21,24-octaoxo-1,4,7,11,14,17,20,23-octazabicyclo[23.3.0]octacosan- } \\
\text { 13-yl]-2-hydroxyacetamide }\end{array}$ & $\begin{array}{c}\text { Kajiyama et } \\
\text { al., } 1998\end{array}$ \\
\hline $\begin{array}{c}\text { Fischerella } \\
\text { muscicola }\end{array}$ & Fischerellin B & $\begin{array}{c}\text { MF: } \mathrm{C}_{20} \mathrm{H}_{29} \mathrm{NO} \\
\text { MW: } 299.4 \mathrm{~g} / \mathrm{mol} \\
\text { IUPAC Name: (3R,5S)-3-methyl-5-[(E)-pentadec-5-en-7,9-diynyl]pyrrolidin-2-one }\end{array}$ & $\begin{array}{c}\text { Srivastava et } \\
\text { al., } 1999\end{array}$ \\
\hline $\begin{array}{l}\text { Lyngbya } \\
\text { majuscula }\end{array}$ & Tanikolide & $\begin{array}{c}\text { MF: } \mathrm{C}_{17} \mathrm{H}_{32} \mathrm{O}_{3} \\
\text { MW: } 284.4 \mathrm{~g} / \mathrm{mol} \\
\text { IUPAC Name: (6R)-6-(hydroxymethyl)-6-undecyloxan-2-one }\end{array}$ & $\begin{array}{c}\text { Singh et al., } \\
1999\end{array}$ \\
\hline $\begin{array}{l}\text { Scytonema } \\
\text { pseudo } \\
\text { hofmanni }\end{array}$ & Scytophycin A & $\begin{array}{c}\text { MF: } \mathrm{C}_{45} \mathrm{H}_{75} \mathrm{NO}_{12} \\
\text { MW: } 822.1 \mathrm{~g} / \mathrm{mol} \\
\text { IUPAC Name: N-[(E,3R,4R,5S,9S,10S,11S)-6,10-dihydroxy-11- } \\
\text { [(1S,3S,4S,5S,7R,8S,9R,12E,14E,17S,19R)-17-hydroxy-3,5,7-trimethoxy-8,14- } \\
\text { dimethyl-11-oxospiro[10,23-dioxabicyclo[17.3.1]tricosa-12,14,20-triene-4,2'- } \\
\text { oxirane]-9-yl]-4-methoxy-3,5,9-trimethyldodec-1-enyl]-N-methylformamide }\end{array}$ & $\begin{array}{l}\text { Matern et } \\
\text { al., } 2001\end{array}$ \\
\hline $\begin{array}{c}\text { Tolypothrix } \\
\text { byssodea }\end{array}$ & Tolybyssidin A & $\begin{array}{c}\text { MF: } \mathrm{C}_{71} \mathrm{H}_{116} \mathrm{~N}_{16} \mathrm{O}_{17} \\
\text { MW: } 1465.8 \mathrm{~g} / \mathrm{mol} \\
\text { IUPAC Name: [(1R)-1-[(3S,6S,9S,12S,15S,18Z,21S,24S,27S,30S,33R,36S,39S)- } \\
\text { 21-benzyl-24,27-bis[(2S)-butan-2-yl]-36-[3-(diaminomethylideneamino)propyl]- } \\
\text { 18-ethylidene-9,30-bis[(1R)-1-hydroxyethyl]-33-(2-methylpropyl)- } \\
\text { 2,5,8,11,14,17,20,23,26,29,32,35,38-tridecaoxo-3,6,15-tri(propan-2-yl)- } \\
\text { 1,4,7,10,13,16,19,22,25,28,31,34,37-tridecazabicyclo[37.3.0]dotetracontan-12- } \\
\text { yl]ethyl] acetate }\end{array}$ & $\begin{array}{c}\text { Jaki et al., } \\
2001\end{array}$ \\
\hline $\begin{array}{c}\text { Tolypothrix } \\
\text { byssodea }\end{array}$ & Tolybyssidin B & $\begin{array}{c}\text { MF: } \mathrm{C}_{72} \mathrm{H}_{114} \mathrm{~N}_{16} \mathrm{O}_{16} \mathrm{~S} \\
\text { MW: } 1491.8 \mathrm{~g} / \mathrm{mol} \\
\text { IUPAC Name: 2-[3-[(2S,5S,8S,11S,14S,17S,20S,23S,26S,29S,32S,35S,38E)-32- } \\
\text { benzyl-17-[(2S)-butan-2-yl]-38-ethylidene-14,20-bis[(1R)-1-hydroxyethyl]-5-[(4- } \\
\text { hydroxyphenyl)methyl]-8-(2-methylsulfanylethyl)- } \\
\text { 3,6,9,12,15,18,21,24,27,30,33,36,39-tridecaoxo-11,23,26,29,35-penta(propan-2-yl)- } \\
\text { 1,4,7,10,13,16,19,22,25,28,31,34,37-tridecazacyclononatriacont-2- } \\
\text { yl]propyl]guanidine }\end{array}$ & $\begin{array}{c}\text { Jaki et al., } \\
2001\end{array}$ \\
\hline $\begin{array}{c}\text { Lyngbya } \\
\text { confervoides }\end{array}$ & Lobocyclamine B & $\begin{array}{c}\text { MF: } \mathrm{C}_{65} \mathrm{H}_{115} \mathrm{~N}_{13} \mathrm{O}_{20} \\
\text { MW: } 1398.7 \mathrm{~g} / \mathrm{mol} \\
\text { IUPAC Name: 3-[(3S,6R,9S,12R,15R,18S,21R,24S,28R,31S,34R,37S,39R)-9- } \\
\text { [(2S)-butan-2-yl]-6-[(1R)-1,2-dihydroxyethyl]-28-heptyl-39-hydroxy-3,31- } \\
\text { bis[(1R)-1-hydroxyethyl]-15,21-bis[(1S)-1-hydroxy-2-methylpropyl]-10,18- } \\
\text { dimethyl-34-(2-methylpropyl)-2,5,8,11,14,17,20,23,26,30,33,36-dodecaoxo-24- } \\
\text { propan-2-yl-1,4,7,10,13,16,19,22,25,29,32,35-dodecazabicyclo[35.3.0]tetracontan- } \\
\text { 12-yl]propanamide }\end{array}$ & $\begin{array}{l}\text { MacMillan } \\
\text { et al., } 2002\end{array}$ \\
\hline $\begin{array}{l}\text { Nostoc } \\
\text { commune }\end{array}$ & Nostodione A & $\begin{array}{c}\text { MF: } \mathrm{C}_{18} \mathrm{H}_{11} \mathrm{NO}_{3} \\
\text { MW: } 289.3 \mathrm{~g} / \mathrm{mol} \\
\text { IUPAC Name: (3E)-3-[(4-hydroxyphenyl)methylidene]-4H-cyclopenta[b]indole- } \\
\text { 1,2-dione }\end{array}$ & $\begin{array}{l}\text { Bhadury \& } \\
\text { Wright, } \\
2004\end{array}$ \\
\hline Hassallia sp. & Hassallidin A & $\begin{array}{c}\text { MF: } \mathrm{C}_{62} \mathrm{H}_{99} \mathrm{~N}_{11} \mathrm{O}_{24} \\
\text { MW: } 1382.5 \mathrm{~g} / \mathrm{mol} \\
\text { IUPAC Name: N-[(2S,3R)-1-[[(3S,6S,12S,15Z,18S,21S,24S,25R)-3,12-bis(3- } \\
\text { amino-3-oxopropyl)-15-ethylidene-21-[(1R)-1-hydroxyethyl]-18-[(4- } \\
\text { hydroxyphenyl)methyl]-7,25-dimethyl-2,5,8,11,14,17,20,23-octaoxo-6-[(1R)-1- } \\
\text { [(2R,3S,4S,5S,6R)-3,4,5-trihydroxy-6-(hydroxymethyl)oxan-2-yl]oxyethyl]-1-oxa- } \\
\text { 4,7,10,13,16,19,22-heptazacyclopentacos-24-yl]amino]-3-hydroxy-1-oxobutan-2- } \\
\text { yl]-2,3-dihydroxytetradecanamide }\end{array}$ & $\begin{array}{l}\text { Neuhof et } \\
\text { al., } 2005\end{array}$ \\
\hline
\end{tabular}

Journal of Experimental Biology and Agricultural Sciences http://www.jebas.org 


\begin{tabular}{|c|c|c|c|}
\hline BGA Spp. & $\begin{array}{l}\text { Antifungal } \\
\text { Compounds }\end{array}$ & Detail of Chemical compounds & References \\
\hline $\begin{array}{l}\text { Fischerella } \\
\text { ambigua }\end{array}$ & $\begin{array}{l}2,4- \\
\text { dichlorobenzoic } \\
\text { acid }\end{array}$ & $\begin{array}{c}\text { MF: } \mathrm{C}_{7} \mathrm{H}_{4} \mathrm{Cl}_{2} \mathrm{O}_{2} \\
\text { MW: } 191.01 \mathrm{~g} / \mathrm{mol} \\
\text { IUPAC Name: 2,4-dichlorobenzoic acid }\end{array}$ & $\begin{array}{l}\text { Wright et } \\
\text { al., } 2005\end{array}$ \\
\hline $\begin{array}{c}\text { Geitlerinema } \\
\text { sp. }\end{array}$ & Swinholide A & $\begin{array}{c}\text { MF: } \mathrm{C}_{78} \mathrm{H}_{132} \mathrm{O}_{20} \\
\text { MW: } 1389.9 \mathrm{~g} / \mathrm{mol} \\
\text { IUPAC }\end{array}$ & $\begin{array}{l}\text { Andrianasol } \\
\text { o et al., } 2005\end{array}$ \\
\hline $\begin{array}{c}\text { Fischerella } \\
\text { ambigua }\end{array}$ & Tjipanazole B & $\begin{array}{c}\text { MF: } \mathrm{C}_{23} \mathrm{H}_{18} \mathrm{Cl}_{2} \mathrm{~N}_{2} \mathrm{O}_{4} \\
\text { MW: } 457.3 \mathrm{~g} / \mathrm{mol} \\
\text { IUPAC Name: (2R,3R,4S,5R)-2-(3,8-dichloro-11H-indolo[2,3-a]carbazol-12- } \\
\text { yl)oxane-3,4,5-triol }\end{array}$ & $\begin{array}{l}\text { Wright et } \\
\text { al., } 2005\end{array}$ \\
\hline $\begin{array}{l}\text { Nadularia } \\
\text { harveyana }\end{array}$ & Norharmane & $\begin{array}{c}\text { MF: } \mathrm{C}_{11} \mathrm{H}_{8} \mathrm{~N}_{2} \\
\text { MW: } 168.19 \mathrm{~g} / \mathrm{mol} \\
\text { IUPAC Name: } 9 \mathrm{H}-\text { pyrido[3,4-b]indole }\end{array}$ & $\begin{array}{l}\text { Volk \& } \\
\text { Furkert, } \\
2006\end{array}$ \\
\hline $\begin{array}{c}\text { Synechocystis } \\
\text { sp. }\end{array}$ & AK-3 & $\begin{array}{c}\text { MF: } \mathrm{C}_{9} \mathrm{H}_{16} \mathrm{~N}_{4} \mathrm{OS} \\
\text { MW: } 228.32 \mathrm{~g} / \mathrm{mol} \\
\text { IUPAC Name: 2-(dimethylamino)-N-(5-propyl-1,3,4-thiadiazol-2-yl)acetamide }\end{array}$ & $\begin{array}{c}\text { Yoon et al., } \\
2006\end{array}$ \\
\hline $\begin{array}{l}\text { Lyngbya } \\
\text { majuscula }\end{array}$ & Hectochlorin & $\begin{array}{c}\text { MF: } \mathrm{C}_{27} \mathrm{H}_{34} \mathrm{Cl}_{2} \mathrm{~N}_{2} \mathrm{O}_{9} \mathrm{~S}_{2} \\
\text { MW: 665.6g/mol } \\
\text { IUPAC Name: [(5S,12S,13S,16S)-12-(4,4-dichloropentyl)-16-(2-hydroxypropan-2- } \\
\text { yl)-4,4,13-trimethyl-2,10,14-trioxo-3,11,15-trioxa-7,18-dithia-20,21- } \\
\text { diazatricyclo[15.2.1.16,9]henicosa-1(19),6(21),8,17(20)-tetraen-5-yl] acetate }\end{array}$ & $\begin{array}{l}\text { Gademann } \\
\text { \& Portmann, } \\
2008\end{array}$ \\
\hline $\begin{array}{l}\text { Calothrix } \\
\text { elenkinii }\end{array}$ & Benzoic Acid & $\begin{array}{c}\text { MF: } \mathrm{C}_{7} \mathrm{H}_{6} \mathrm{O}_{2} \\
\text { MW: } 122.12 \mathrm{~g} / \mathrm{mol} \\
\text { IUPAC Name: benzoic acid }\end{array}$ & $\begin{array}{c}\text { Natarajan et } \\
\text { al., } 2012\end{array}$ \\
\hline
\end{tabular}

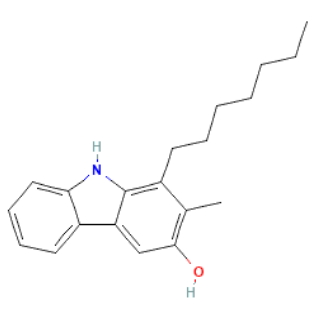

Carazostatin

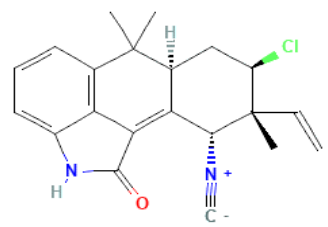

Anhydrohaloxindole A; Anhydrohapaloxindole A

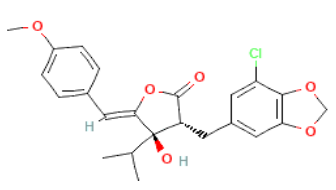

Cyanobacterin

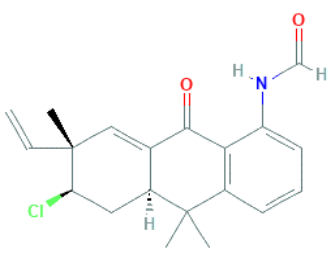

Fontonamide

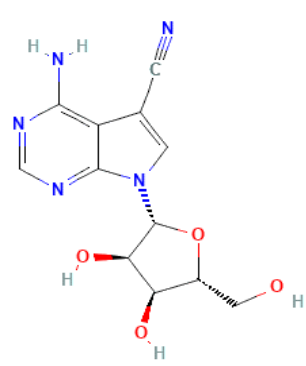

Toyocamycin

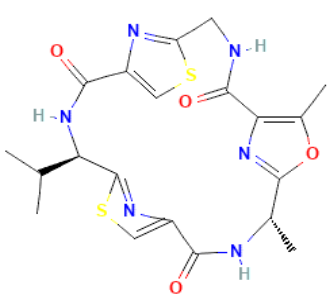

Nostocyclamide

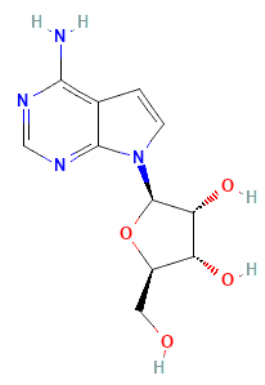

Tubercidin

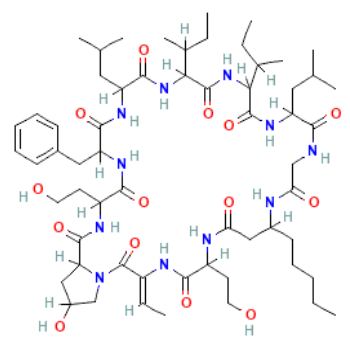

Hormothamnin A

Journal of Experimental Biology and Agricultural Sciences http://www.jebas.org 


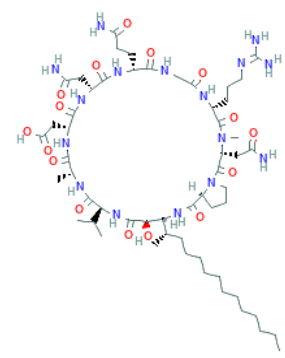

Calophycin

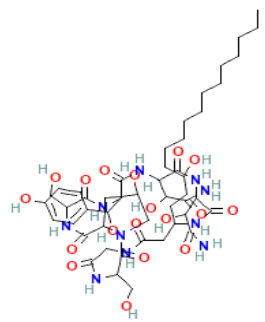

Nostofungicidine

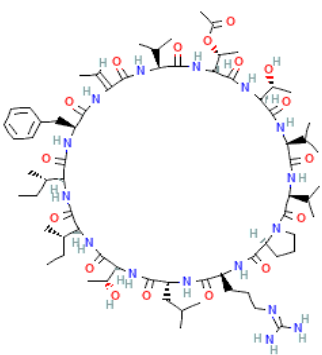

Tolybyssidin A

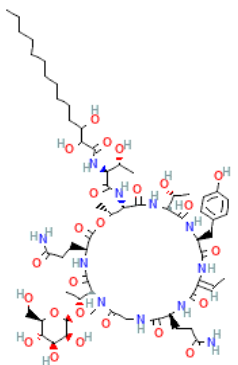

Hassallidin A

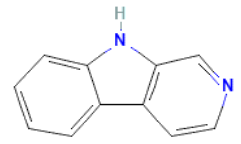

Norharmane

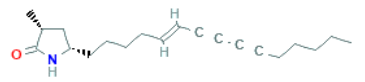

Fischerellin B

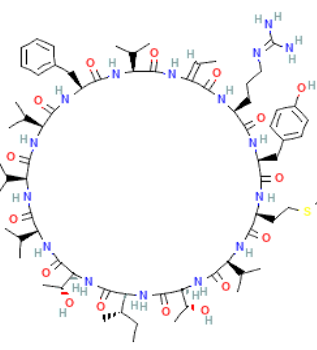

Tolybyssidin B<smiles>O=C(O)c1ccc(Cl)cc1Cl</smiles>

2,4-dichlorobenzoic acid

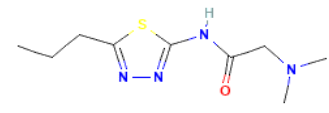

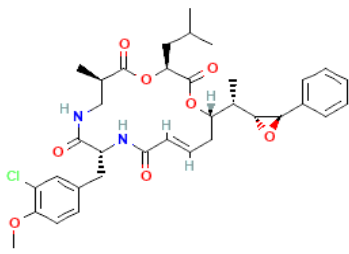

Cryptophycin 1

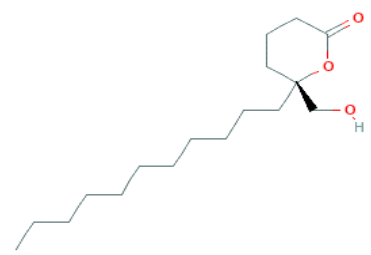

Tanikolide

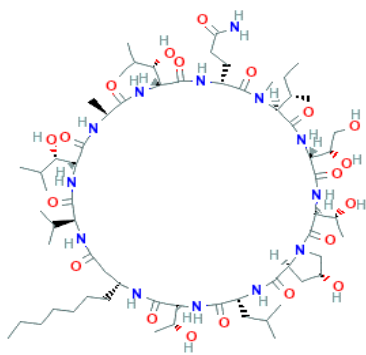

Lobocyclamine B

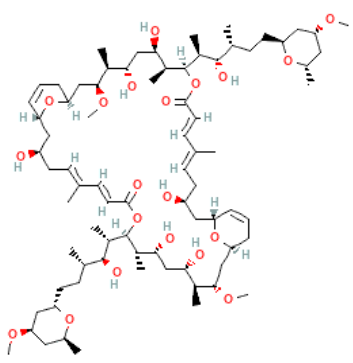

Swinholide A

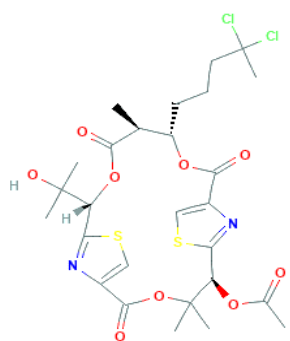

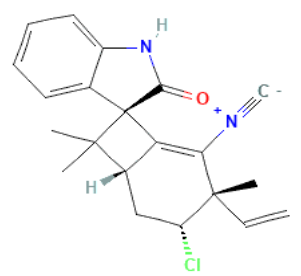

Welwitindolinone A isonitrile

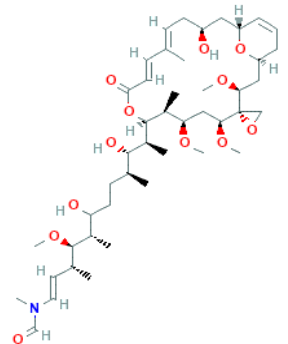

Scytophycin A

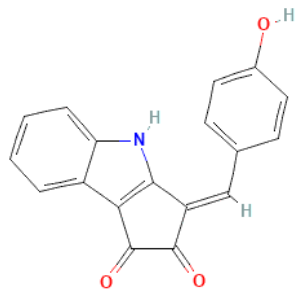

Nostodione A

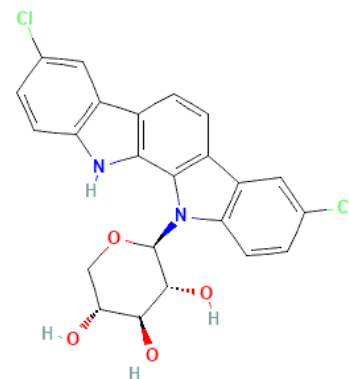

Tjipanazole B

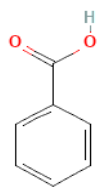

Benzoic Acid

Figure 13 Chemical structures of the active ingredients isolated from various BGA and having antifungal propeties 
Table 4 Antioxidants compounds recently purified from Blue-Green Algae

\begin{tabular}{|ccc|}
\hline Antioxidants compounds & Source & References \\
\hline Pyrogallol, E-Vanillic, Hespirdin & Spirulina platensis & Gabr et al., 2020 \\
\hline Benzeneacetanomide and Norvaline, n-propargyloxycarbonyl & Microcystis aeruginosa & $\begin{array}{c}\text { Vasudevan et al., } \\
2020\end{array}$ \\
\hline BHA, Beta tocopherol, Phytosterols & Spirulina maxima & Gamal et al., 2020 \\
\hline $\begin{array}{c}\text { Caffeic acid, syringic acid, ferulic acid, p-coumaric acid, chlorogenic acid, } \\
\text { kaempferol, quercetin and apigenin } \gamma \text {-linolenic acid, } \alpha \text {-linolenic acid }\end{array}$ & Spirulina platensis & Bellahcen et al., 2020 \\
\hline $\begin{array}{c}\text { Benzoic acid, 4-(1-azepinyl)azo-, ethyl ester (b) 1,6-methanonaphthalen-1(2H)-ol, } \\
\text { octahydro-4,8a,9,9-tetramethyl, (c) Dibutyl phthalate, (d) 1,2-benzene dicarboxylic } \\
\text { acid, (e) Hexadecanoic acid, 2-pentadecyl-1,3-dioxan-5-yl ester }\end{array}$ & Oscillatoria sp. SSCM01 & Nainangu et al., 2020 \\
\hline
\end{tabular}

Table 5 Types of BGA Extracts, Assay methods and their antioxidant potential

\begin{tabular}{|c|c|c|c|c|}
\hline Blue-Green Algae & Type of Extract & Assay methods & $\begin{array}{l}\text { Maximum } \\
\text { Activity }\end{array}$ & References \\
\hline Spirulina platensis & $\begin{array}{l}\text { Ethanolic and } \\
\text { aqueous extract }\end{array}$ & $\begin{array}{c}\text { DPPH radical- } \\
\text { scavenging Assay }\end{array}$ & $96.33 \%$ & Gabr et al., 2020 \\
\hline Microcystis aeruginosa & Methanol Extract & $\begin{array}{l}\text { Scavenging ability on 1, 1-diphenyl- } \\
\text { 2-picrylhydrazyl radicals (DPPH) } \\
\text { Hydroxyl radical scavenging assay }\end{array}$ & $\begin{array}{l}54 \% \\
49 \%\end{array}$ & $\begin{array}{l}\text { Vasudevan et al., } \\
\qquad 2020\end{array}$ \\
\hline Spirulina maxima & - & $\begin{array}{c}\text { DPPH radical- } \\
\text { scavenging Assay }\end{array}$ & $25.73 \%$ & Gamal et al., 2020 \\
\hline Spirulina platensis & $\begin{array}{l}\text { Ethanolic, aqueous, } \\
\text { and lipidic extracts }\end{array}$ & $\begin{array}{l}\text { Diphenyl-1-picrylhydrazyl (DPPH) } \\
\text { Azino-bis (ethylbenzthiazoline-6- } \\
\text { sulfonicacid (ABTS) }\end{array}$ & $\begin{array}{l}(\mathrm{IC} 50=449 \mu \mathrm{g} / \mathrm{mL} \pm 83) \\
(\mathrm{IC} 50=740 \mu \mathrm{g} / \mathrm{mL} \pm 12)\end{array}$ & $\begin{array}{l}\text { Bellahcen et al., } \\
2020\end{array}$ \\
\hline $\begin{array}{l}\text { Oscillatoria sp. } \\
\text { SSCM01 }\end{array}$ & $\begin{array}{c}\mathrm{MeOH}: \mathrm{CHCl} 3 \\
\text { fraction }\end{array}$ & DPPH radical scavenging assay & $48 \%$ & $\begin{array}{c}\text { Nainangu et al., } \\
2020\end{array}$ \\
\hline Spirulina platensis & crude extracts & DPPH radical-scavenging activity & $45.75 \%$ & Safari et al., 2020 \\
\hline Oscillatoria acuminate & Methanolic extract & $\begin{array}{c}\text { DPPH (2, 2- diphenyl-1- } \\
\text { picrylhydrazyl) } \\
\text { ABTS }^{+}(2,2 \text {-azino-bis (3- } \\
\text { ethylbenzthiazoline-6-sulfonic acid) }\end{array}$ & $\begin{array}{l}6.58 \% \\
34.60 \%\end{array}$ & $\begin{array}{l}\text { Gheda \& Ismail } \\
\text { (2020). }\end{array}$ \\
\hline \multirow[b]{2}{*}{$\begin{array}{l}\text { Dolichospermum flos- } \\
\text { aquae HSSASE2 }\end{array}$} & & DPPH radical scavenging assay & $(467.7 \mu \mathrm{g} / \mathrm{ml})$ & \\
\hline & & $\begin{array}{c}\text { Nitric oxide radical scavenging assay } \\
\text { Anti-lipid peroxidation assay }\end{array}$ & $\begin{array}{c}(\mathrm{IC} 50=28.7 \pm 0.1 \\
\mu \mathrm{g} / \mathrm{ml}) \\
(\mathrm{IC} 5011.9 \pm 0.2 \\
\mu \mathrm{g} / \mathrm{ml})\end{array}$ & Senousy et al., 2020 \\
\hline $\begin{array}{l}\text { Anabaena } \mathrm{sp} . \\
\text { Stigonaema } \text { sp. and } \\
\text { Oscillatoria } \mathrm{sp} .\end{array}$ & Methanol Extract & $\begin{array}{l}\text { Total Antioxidant capacity } \\
\text { determination kit }\end{array}$ & $\begin{array}{c}0.346(\mathrm{mM} / \mathrm{L}) ; 0.36 \\
(\mathrm{mM} / \mathrm{L}) \text { and } 0.37 \\
(\mathrm{mM} / \mathrm{L})\end{array}$ & Seddek et al., 2019 \\
\hline $\begin{array}{c}\text { Aphanizomenon } \\
\text { gracile (LMECYA } \\
\text { 009), Aphanizomenon } \\
\text { flos-aquae (LMECYA } \\
\text { 088), Nostoc } \\
\text { (LMECYA 291), } \\
\text { Plankto thrixmougeotii } \\
\text { (LEGE 06224) }\end{array}$ & $\begin{array}{l}\text { Methanolic and } \\
\text { ethanolic }\end{array}$ & $\begin{array}{l}\text { DPPH scavenging method, } \\
\beta \text {-carotene bleaching assay }\end{array}$ & $\begin{array}{c}10.7 \% \\
828.94 \mathrm{AAC}\end{array}$ & $\begin{array}{l}\text { Guerreiro et al., } \\
2019\end{array}$ \\
\hline
\end{tabular}

Journal of Experimental Biology and Agricultural Sciences http://www.jebas.org 


\subsubsection{Anti-cancer potential of BGA}

BGA extracts and compounds are known to exhibit anticancer properties (Shishido et al., 2020; Gara-Ali et al., 2021). Research by Jaspers \& Lawton (1998) has focused on various biologically active compounds from BGA. The curian A, a novel lipid compound isolated from Lyngbya majuscula, is a potent inhibitor of microtubule assembly with very low $I C_{50}$ values against L1210 leukemia cells and CD-46 Burkitt lymphoa cells, at par with those for colchicines. Cryptophycin 1 and 8 , another anticancer compound was first isolated from Nostoc sp. by researchers at Merck. The oral supplement of Spirulina fusiformis is known for regression of subjects with homogenous leukolakia (Mathew et al., 1995). The extracts of Spirulina and Dunaliella inhibited the chemically induced carcinogenesis in model hamster buccal pouches (Schwartz et al., 1988). Studies have also shown that sulphated polysachharide, calcium spirulans appears to inhibit tumor invasion of melanoma cells and basement membrane
(Mishima et al., 1998). Aphanizomenon flosaquae extract containing a high concentration of phycocyanin inhibited the in vitro growth of tumour cells, indicating the sensitivity of cell lines to the phycocyanin. A filamentous cyanobacterium Phormidium tenue contains several diacylglycerols that inhibit chemically induce tumors on mice (Tokuda et al., 1996). Similarly, cryprophycin 1 isolated from Nostoc sp. (ATCC 53789) is the most potent suppressor of microtubule dynamics i.e. it blocks all cell cycles in G2/M phase. Curacin A is isolated from Lyngbya majuscule. This compound is found to be a potent inhibitor of microtubule assembly. There is a need for immediate attention for more novel anticancer drugs so that carcinogenic cells are capable of resisting some drugs, like vinca alkaloids and taxanes. These drugs failed to treat cancer in a chemotherapeutic way. Cancer is known to be the major cause of mortality worldwide. Recently some new types of cancer e.g. gliobastoma are increased rapidly. Anticancer compounds reported from BGA are summarized in Table 6 Figure 14.

Table 6 Anticancer compounds reported from BGA

\begin{tabular}{|c|c|c|c|}
\hline BGA Spp. & $\begin{array}{l}\text { Anticancer } \\
\text { Compounds }\end{array}$ & Chemical Structure & References \\
\hline Calothrix sp. & Calothrixin A & $\begin{array}{c}\text { MF: } \mathrm{C}_{19} \mathrm{H}_{10} \mathrm{~N}_{2} \mathrm{O}_{3} \\
\text { MW: } 314.3 \mathrm{~g} / \mathrm{mol} \\
\text { IUPAC Name: } 20 \text {-oxido-10-aza-20- } \\
\text { azoniapentacyclo[11.8.0.03,11.04,9.014,19]henicosa-1(13),3(11),4,6,8,14,16,18,20- } \\
\text { nonaene-2,12-dione }\end{array}$ & $\begin{array}{c}\text { Cardellina et } \\
\text { al., 1979b }\end{array}$ \\
\hline Nostoc sp. & Boromycin & $\begin{array}{c}\text { MF: } \mathrm{C}_{45} \mathrm{H}_{74} \mathrm{BNO}_{15} \\
\text { MW: } 879.9 \mathrm{~g} / \mathrm{mol} \\
\text { IUPAC Name: }[(2 \mathrm{R})-1-[(1 \mathrm{R})-1- \\
{[(1 \mathrm{R}, 5 \mathrm{~S}, 7 \mathrm{E}, 11 \mathrm{~S}, 13 \mathrm{~S}, 16 \mathrm{R}, 17 \mathrm{R}, 24 \mathrm{~S}, 25 \mathrm{R}, 27 \mathrm{R}, 31 \mathrm{R}, 33 \mathrm{~S}, 36 \mathrm{R})-11,31 \text {-dihydroxy- }} \\
\text { 12,12,16,25,32,32,36-heptamethyl-3,22-dioxo-4,18,20,23,26,37,38,40,41-nonaoxa-19- } \\
\text { boranuidaheptacyclo[17.17.1.11,33.12,19.113,17.124,27.017,21]hentetracont-7-en-5- } \\
\text { yl]ethoxy]-3-methyl-1-oxobutan-2-yl]azanium }\end{array}$ & $\begin{array}{c}\text { Banker \& } \\
\text { Carmeli, } \\
\text { 1998; Gupta, } \\
2012\end{array}$ \\
\hline $\begin{array}{l}\text { Scytonema } \\
\text { varium }\end{array}$ & Scytovirin & $\begin{array}{c}\text { MF: } \mathrm{C}_{66} \mathrm{H}_{91} \mathrm{~N}_{19} \mathrm{O}_{24} \mathrm{~S} \\
\text { MW: } 1566.6 \mathrm{~g} / \mathrm{mol} \\
\text { IUPAC Name: (4S)-4-[[(2S)-4-amino-2-[[(2S)-2-[[(2R)-2-[[(2S)-2-[[(2S,3R)-2-[[(2S)- } \\
\text { 1-[2-[[(2S)-2-[(2-aminoacetyl)amino]-3-hydroxypropanoyl]amino]acetyl]pyrrolidine- } \\
\text { 2-carbonyl]amino]-3-hydroxybutanoyl]amino]-3-(4-hydroxyphenyl)propanoyl]amino]- } \\
\text { 3-sulfanylpropanoyl]amino]-3-(1H-indol-3-yl)propanoyl]amino]-4- } \\
\text { oxobutanoyl]amino]-5-[[(2S)-1-[[(2S)-4-amino-1-[[(2S)-4-amino-1-[(2S)-2- } \\
\text { (carboxymethylcarbamoyl)pyrrolidin-1-yl]-1,4-dioxobutan-2-yl]amino]-1,4- } \\
\text { dioxobutan-2-yl]amino]-1-oxopropan-2-yl]amino]-5-oxopentanoic acid }\end{array}$ & $\begin{array}{c}\text { Shi et al., } \\
1999\end{array}$ \\
\hline $\begin{array}{c}\text { Symploca } \\
\text { genus }\end{array}$ & Largazole & $\begin{array}{c}\text { MF: } \mathrm{C}_{29} \mathrm{H}_{42} \mathrm{~N}_{4} \mathrm{O}_{5} \mathrm{~S}_{3} \\
\text { MW: 622.9g/mol } \\
\text { IUPAC Name: S-[(E)-4-[(5R,8S,11S)-5-methyl-6,9,13-trioxo-8-propan-2-yl-10-oxa- } \\
\text { 3,17-dithia-7,14,19,20-tetrazatricyclo[14.2.1.12,5]icosa-1(18),2(20),16(19)-trien-11- } \\
\text { yl]but-3-enyl] octanethioate }\end{array}$ & $\begin{array}{l}\text { Luesch et al. } \\
2001\end{array}$ \\
\hline Nostoc sp. & Apratoxin A & $\begin{array}{c}\text { MF: } \mathrm{C}_{45} \mathrm{H}_{69} \mathrm{~N}_{5} \mathrm{O}_{8} \mathrm{~S} \\
\text { MW: } 840.1 \mathrm{~g} / \mathrm{mol} \\
\text { IUPAC Name: (2S,3S,5S,7S,10S,16S,19S,22S,25E,27S)-16-[(2S)-butan-2-yl]-7-tert- } \\
\text { butyl-3-hydroxy-22-[(4-methoxyphenyl)methyl]-2,5,17,19,20,25-hexamethyl-8-oxa- } \\
\text { 29-thia-14,17,20,23,30-pentazatricyclo[25.2.1.010,14]triaconta-1(30),25-diene- } \\
9,15,18,21,24 \text {-pentone }\end{array}$ & $\begin{array}{c}\text { Grinberg et } \\
\text { al., } 2002\end{array}$ \\
\hline
\end{tabular}

Journal of Experimental Biology and Agricultural Sciences http://www.jebas.org 


\begin{tabular}{|c|c|c|c|}
\hline BGA Spp. & $\begin{array}{l}\text { Anticancer } \\
\text { Compounds }\end{array}$ & Chemical Structure & References \\
\hline $\begin{array}{c}\text { Dolabella } \\
\text { auricularia }\end{array}$ & Dolastatin 15 & $\begin{array}{c}\text { MF: } \mathrm{C}_{45} \mathrm{H}_{68} \mathrm{~N}_{6} \mathrm{O}_{9} \\
\text { MW: } 837.1 \mathrm{~g} / \mathrm{mol} \\
\text { IUPAC Name: [(2S)-1-[(2S)-2-benzyl-3-methoxy-5-oxo-2H-pyrrol-1-yl]-3-methyl-1- } \\
\text { oxobutan-2-yl] (2S)-1-[(2S)-1-[(2S)-2-[[(2S)-2-[[(2S)-2-(dimethylamino)-3- } \\
\text { methylbutanoyl]amino]-3-methylbutanoyl]-methylamino]-3- } \\
\text { methylbutanoyl]pyrrolidine-2-carbonyl]pyrrolidine-2-carboxylate }\end{array}$ & $\begin{array}{c}\text { Stevenson et } \\
\text { al., } 2002\end{array}$ \\
\hline Cyanobacteria & Astaxanthin & - & $\begin{array}{c}\text { Chen et al., } \\
2003\end{array}$ \\
\hline Nostoc sp. & Cryptophycin & $\begin{array}{c}\text { MF: } \mathrm{C}_{35} \mathrm{H}_{43} \mathrm{ClN}_{2} \mathrm{O}_{8} \\
\text { MW: } 655.2 \mathrm{~g} / \mathrm{mol} \\
\text { IUPAC Name: (3S,6R,10R,13E,16S)-10-[(3-chloro-4-methoxyphenyl)methyl]-6- } \\
\text { methyl-3-(2-methylpropyl)-16-[(1S)-1-[(2R,3R)-3-phenyloxiran-2-yl]ethyl]-1,4- } \\
\text { dioxa-8,11-diazacyclohexadec-13-ene-2,5,9,12-tetrone }\end{array}$ & $\begin{array}{c}\text { Back \& } \\
\text { Liang 2005; } \\
\text { Medina et } \\
\text { al., } 2008\end{array}$ \\
\hline L. majusculata & Curacin A & 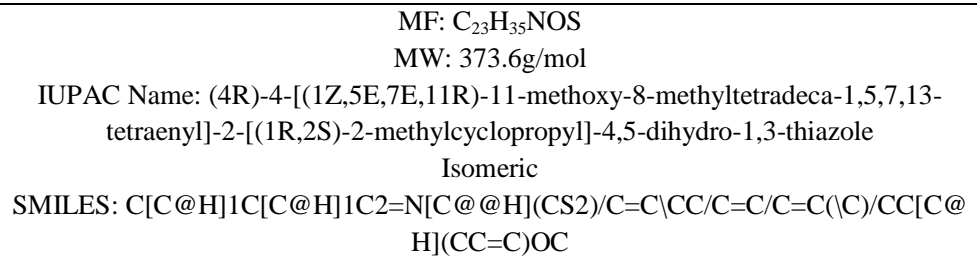 & $\begin{array}{c}\text { Xiong et al., } \\
2006\end{array}$ \\
\hline Lyngbya sp. & Dragonamide C & $\begin{array}{c}\text { MF: } \mathrm{C}_{33} \mathrm{H}_{57} \mathrm{~N}_{5} \mathrm{O}_{6} \\
\text { MW: } 619.8 \mathrm{~g} / \mathrm{mol} \\
\text { IUPAC Name: (E)-N-[(2S)-1-[[(2S)-1-[[(2S)-1-[[(2S)-1-amino-3-methyl-1-oxobutan- } \\
\text { 2-yl]-methylamino]-3-methyl-1-oxobutan-2-yl]-methylamino]-3-methyl-1-oxobutan- } \\
\text { 2-yl]-methylamino]-3-methyl-1-oxobutan-2-yl]-3-methoxy-N-methyloct-2-en-7- } \\
\text { ynamide }\end{array}$ & $\begin{array}{l}\text { Gunasekera } \\
\text { et al., } 2008\end{array}$ \\
\hline Lyngbya sp. & Dragonamide D & $\begin{array}{c}\text { MF: } \mathrm{C}_{32} \mathrm{H}_{55} \mathrm{~N}_{5} \mathrm{O}_{6} \\
\mathrm{MW}: 605.8 \mathrm{~g} / \mathrm{mol} \\
\text { IUPAC Name: N-[(2S)-1-[[(2S)-1-[[(2S)-1-[[(2S)-1-amino-3-methyl-1-oxobutan-2- } \\
\text { yl]-methylamino]-3-methyl-1-oxobutan-2-yl]-methylamino]-3-methyl-1-oxobutan-2- } \\
\text { yl]-methylamino]-3-methyl-1-oxobutan-2-yl]-N-methyl-3-oxooct-7-ynamide }\end{array}$ & $\begin{array}{l}\text { Gunasekera } \\
\text { et al., } 2008\end{array}$ \\
\hline
\end{tabular}

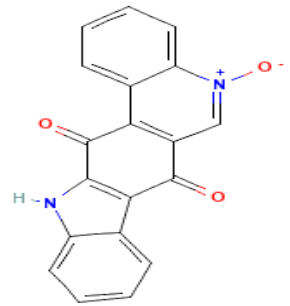

Calothrixin A

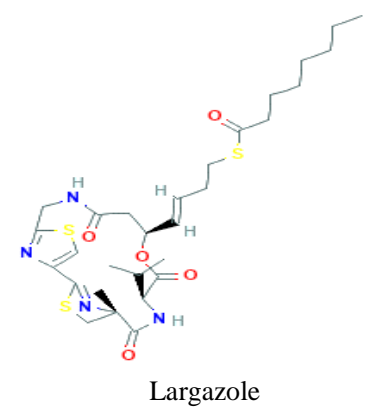

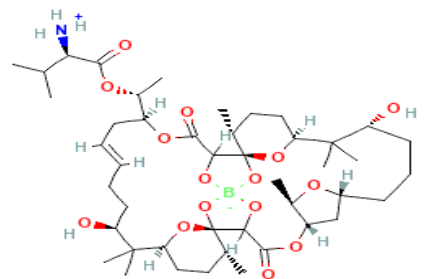

Boromycin

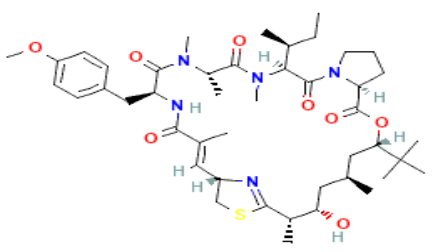

Apratoxin A

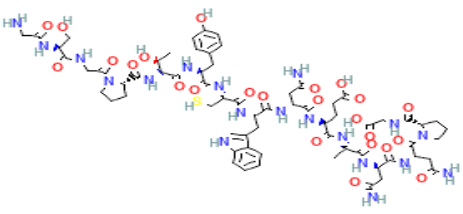

Scytovirin

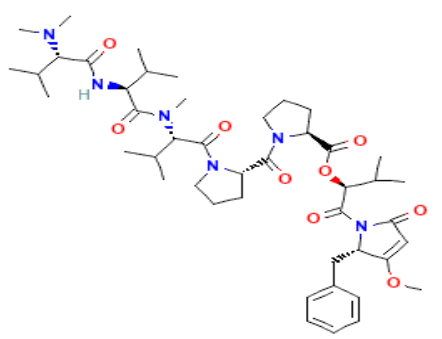

Dolastatin 15

Journal of Experimental Biology and Agricultural Sciences http://www.jebas.org 


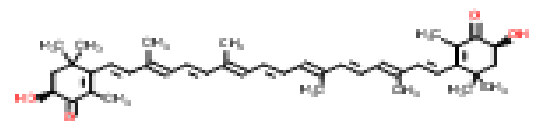

Astaxanthin

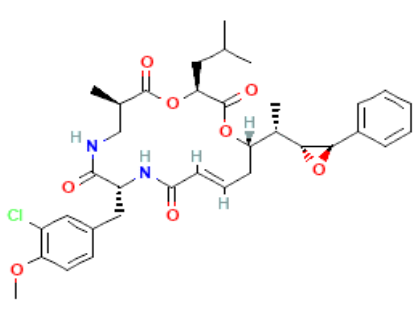

Cryptophycin

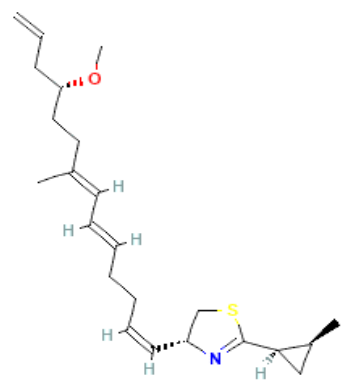

Curacin A

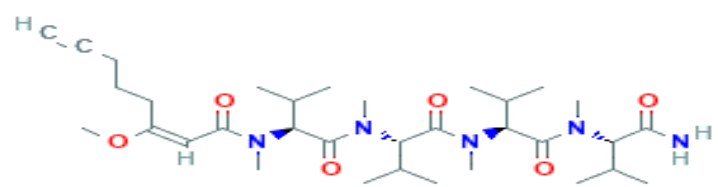

Dragonamide C

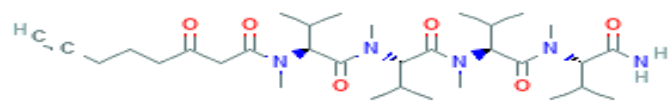

Dragonamide D

Figure 14 Chemical structures of the active ingredients isolated from various BGA and having anticancerous properties

\subsubsection{Antiviral Potential including SARS-CoV-2}

The globe is so much affected by the dreadful diseases caused by infection of viruses such as HIV-acquired immune deficiency syndrome. There is also another viral deadly disease that is dengue which may have many consequences. Despite two former major outbreaks of coronavirus infections i.e. the SARS and MERS, the world is still underprepared to effectively manage the current COVID-19 pandemic outbreak. The researchers were in search of a novel and potent drug which will be able to resist those deadly and dreadful viral infections throughout the world. Scientists have now invented novel, potent, and safe anti-viral agents that are very useful in this urgent situation. Recently there is a new scientific treatment or therapy which is named highly active antiretroviral therapy in short HAART. This is triple therapy which is very fruitful and capable in the treatment of HIV infections which is very helpful and makes control and resistance power in carcinogenic treatment. This therapy can create strong viral resistance. But this therapy cannot stop the viral agent which is causing such kinds of issues. BGA species are also known to produce substances that have been proved to be anti-HIV, therefore can be exploited in therapy against AIDS (Schaeffer \& Krylov, 2000; Carpine \& Sieber, 2021)
Gustafson et al. (1989) used a tetrazolum-based micro-culture to screen extracts of cultured marine cyanobacteria, Lyngbyalager heimii, and Phormedium tenue, for the inhibition of HIV-1. This led to the discovery of sulfonic acid containing glycolipids as a new class of HIV-1- inhibitory compounds. Other cyanobacteria, Phormedium cebennse, Oscillatoria raciborskii, Scytonem aburmanicum, Calothrix elenkinii, and Anabaena variabilis, gave extracts that inhibited HIV-1 and gave positive tests for the presence of sulfolipids.

Compounds and extracts with anti-HIV activity are also active against other retroviruses such as Herpes simplex virus (HSV) and respiratory syncytial virus, but the amount of antiviral activity varies with the compound and the virus. Most of the research has focused on sulphated homopolysaccharides and heteropolysaccharides, sulfoglycolipids, carrageenans, fucoidan, sesquiterpene hydroquinones, and other classes of compounds with an anti-HIV activity that has been isolated from algae have received less attention. Hayashi et al. (1996) isolated calcium spirulan, a sulfated polysaccharide obtained from a marine bluegreen alga, Spirulina platensis which inhibited the Herpes simplex virus. Subsequently, Ayehunie et al. (1998) determined that an aqueous extract of $S$. platensis, at a concentration that was nontoxic to human cells, inhibited syncytium formation and HIV-1 
replication in human T-cell lines, peripheral blood mononuclear cells, and Langerhans cells. The antiviral effects of polysaccharides from marine algae towards mumps virus and influenza B virus were reported by Gerber et al. (1958). Subsequently, polysaccharides fractions from extracts of red algae were found to inhibit the herpes simplex virus (HSV). Similarly, Boyd et al. (1997) isolated Cyanovirin-N from an aqueous cellular extract of cyanobacterium Nostoc ellipsosporum which has been proved to be antiviral. Lau et al., (1993) reported that the lipophilic and hydrophilic extracts of over 900 strains of cultured blue-green algae in vitro for their ability to inhibit the reverse transcriptases of avian myeloblastosis virus (Table 7)

Various compounds have been isolated from a variety of bluegreen algaeBGA-derived polysaccharides that have been reported for the inhibition of SARS-CoV-2 (Sami et al., 2021). Few organizations are actively involved in developing algae-based edible vaccines for SARS-CoV-2 (Jafari et al., 2021) (Table 8)

\subsubsection{Antidiabetic Potential of BGA}

Blue-green algae are known to exhibit potential antidiabetic properties. In a study conducted by Priatni et al. (2016) methanol extract of marine cyanobacterial strains such as Oscillatoria limnetica, Coelastrella sp., Oscillatoria sp., Chroococcus sp., Leptolyngbya sp., Pseudanabaena sp., Lyngbya sp., Aphanothece sp., Phormidiumsp, and Synechococcus sp. have potential antidiabetic potential. The metabolites of Pseudanabaena sp. showed the highest $\alpha$ glucosidase inhibition. In another study, Egyptian Scientists evaluated extracts of Fischerella sp. BS1-EG for antidiabetic

Table 7 Antiviral Compounds reported from BGA

\begin{tabular}{|c|c|c|c|}
\hline BGA Sp. & Anti Viral Compound & Chemical Structure & References \\
\hline $\begin{array}{c}\text { Nostoc } \\
\text { ellipsosporum }\end{array}$ & Cyanovirin-N & 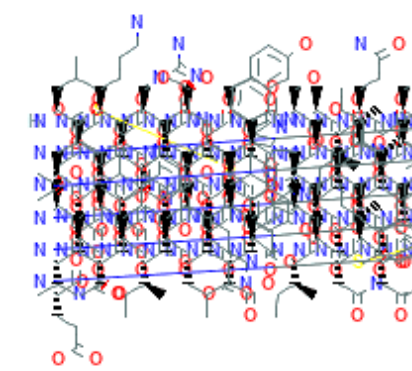 & $\begin{array}{c}\text { Boyd et al., } \\
\text { 1997;; } \\
\text { Bewley, } 2001\end{array}$ \\
\hline $\begin{array}{l}\text { Microcystis } \\
\text { aeruginosa }\end{array}$ & Microvirin & & $\begin{array}{c}\text { Kehr et al., } \\
2006\end{array}$ \\
\hline $\begin{array}{c}\text { Scytonema } \\
\text { varium }\end{array}$ & $\begin{array}{c}\text { Scytovirin } \\
\text { MF: } \mathrm{C}_{66} \mathrm{H}_{91} \mathrm{~N}_{19} \mathrm{O}_{24} \mathrm{~S} \\
\text { MW: } 1566.6 \mathrm{~g} / \mathrm{mol} \\
\text { IUPAC Name: (4S)-4-[[(2S)-4-amino-2- } \\
\text { [[(2S)-2-[[(2R)-2-[[(2S)-2-[[(2S,3R)-2- } \\
\text { [[(2S)-1-[2-[[(2S)-2-[(2- } \\
\text { aminoacetyl)amino]-3- } \\
\text { hydroxypropanoyl]amino]acetyl]pyrrolidine- } \\
\text { 2-carbonyl]amino]-3- } \\
\text { hydroxybutanoyl]amino]-3-(4- } \\
\text { hydroxyphenyl)propanoyl]amino]-3- } \\
\text { sulfanylpropanoyl]amino]-3-(1H-indol-3- } \\
\text { yl)propanoyl]amino]-4-oxobutanoyl]amino]- } \\
\text { 5-[[(2S)-1-[[(2S)-4-amino-1-[[(2S)-4-amino- } \\
\text { 1-[(2S)-2- } \\
\text { (carboxymethylcarbamoyl)pyrrolidin-1-yl]- } \\
\text { 1,4-dioxobutan-2-yl]amino]-1,4-dioxobutan- } \\
\text { 2-yl]amino]-1-oxopropan-2-yl]amino]-5- } \\
\text { oxopentanoic acid }\end{array}$ & $\underbrace{N_{N^{H}}}_{H_{N}^{N}}$ & $\begin{array}{c}\text { Bokesch et } \\
\text { al., } 2003\end{array}$ \\
\hline $\begin{array}{l}\text { M. viridis } \\
\text { NIES-02 }\end{array}$ & Microcystis Viridis Lectin & NA & $\begin{array}{l}\text { Yamaguchi } \\
\text { et al., } 1999\end{array}$ \\
\hline $\begin{array}{c}\text { Arthrospira } \\
\text { platensis }\end{array}$ & Calcium Spirulan & NA & $\begin{array}{c}\text { Hayashi et } \\
\text { al., } 1996\end{array}$ \\
\hline
\end{tabular}

Journal of Experimental Biology and Agricultural Sciences http://www.jebas.org 
Table 8 Anti-HIV activity of compounds from BGA

\begin{tabular}{|c|c|c|}
\hline BGA strains & Compounds & References \\
\hline $\begin{array}{c}\text { Anabaena variabilis, Calothrix elenkinii, Lyngbya } \\
\text { lagerheimii, Phormidium tenue, Phormedium } \\
\text { cebennse, Oscillatoria raciborskii, Scytonema } \\
\text { burmanicum }\end{array}$ & $\begin{array}{l}\text { Extracts containing sulfolipids (sulfoquinovosyl } \\
\text { diacylglycerols) }\end{array}$ & Gustafson et al., 1989 \\
\hline Spirulina platensis & activity in polysaccharide fraction & Ayehunie et al., 1998 \\
\hline Spirulina platensis (Marine) & $\begin{array}{c}\text { Calcium spirulan, Ca-SP } \\
\text { (sulfated polysaccharide fraction) Dextran sulfate }\end{array}$ & Hayashi et al., 1996 \\
\hline Nostoc ellipsosporum & Cyanovirin- $\mathrm{N}$ (11-k Da antiviral protein & Gustafson et al., 1996 \\
\hline Nostoc ellipsosporum & Cyanovirin- $\mathrm{N}$ (11-k Da antiviral protein & Boyd et al., 1996 \\
\hline 900 strains & Lipophilic and Hydrophilic extracts & Lau et al., 1993 \\
\hline Scytonema spp. & Sulfoglycolipids & Reshef et al., 1997 \\
\hline Oscillatoria spp. & Acylated diglycolipids & Reshef et al., 1997 \\
\hline $\begin{array}{l}\text { Oscillatoria raoi(TAU IL-76-12), Syctonema spp. } \\
\text { (TAU SL-30-1-4), Oscillatoria trichoides(TAU } \\
\text { IL 104-3-2), Phormidium tenue ( TAU IL-144-1), } \\
\text { Oscillatoria limnetica, Lammermann (TAU NG- } \\
\text { 4-1-2) }\end{array}$ & $\begin{array}{l}\text { Sulfolipids, Sulfoglycolipids, hydrolysis products, } \\
\text { synthetic derivative }\end{array}$ & Loya et al., 1998 \\
\hline
\end{tabular}<smiles>COC(=O)c1ccc2c(c1)C[C@]1(C)[C@@H](C)CC[C@@]3(C)[C@@]1(C)[C@@H](O)C[C@H](O)[C@@]23C</smiles>

Figure 15 Chemical structure of Anti-inflammatory compounds Tolypodiol isolated from Tolypothrix nodosa

potential and have reported certain bioactive compounds responsible for the activity (Ahmed et al., 2018). In a recent study, Sridhar et al. (2021) have evaluated phycocyanin of $S$. platensis for its antidiabetic potential by assessing $\alpha$-amylase and $\beta$-glucosidase enzyme inhibition using spectroscopy techniques. In this in vitro test, significant Antidiabetic activity (88\%) was observed at a concentration of $250 \mu \mathrm{g} / \mathrm{ml}$. In some studies, lesser antidiabetic properties have been reported, like Ghosh et al. (2016) evaluated in vitro antidiabetic properties of molecules from Lyngbya, Microcoleus, and Synechocystis sp. by $\alpha$-amylase inhibition method and stated the lesser enzyme inhibition effect. Similarly, Xu et al. (2012) described the lowest $\alpha$-amylase enzyme inhibition activity of Phlorotannins pigments extracted from Eckloniakurome. However, Hwang et al. (2014) reported $65-80 \%$ inhibitory activity at the concentration of $250 \mu \mathrm{g} / \mathrm{ml}$ while this was reported $51-67 \%$ at the dose of $200 \mu \mathrm{g} / \mathrm{ml}$ dose of S. platensis phycocyanin. Lesser enzyme inhibition even in higher concentrations was reported in a study conducted by Priatni et al. (2016).

\subsubsection{Anti-inflammatory Activity of BGA}

Blue-Green Algae contain a significant amount of carotenoids i.e. $\beta$-carotene, lycopene, lutein having antioxidant properties. By the quenching action on the reactive oxygen species, these carotenoids also have anti-inflammatory activity. This anti-inflammatory activity might be due to the presence of phycocyanin, a photo harvesting pigment. Further, the anti-inflammatory effect seemed to be the result of leucotriene formation inhibition by phycocyanin, an inflammatory metabolite of arachidonic acid (Romay et al., 1999). Apbanizomenon flosaquae decrease the level of arachidonic acid. Further, A. flosaquae and Spirulina contain significant amount of omega-3-alpha linolenic acid which inhibits the formation of inflammatory prostaglandins and arachidonate metabolites (Figure 15).

\section{Conclusion and Future possibilities}

BGA are groups of extraordinary, diverse, gram-negative, oxygenic, photosynthetic prokaryotic microscopic organisms. 
Blue-green algae are found all over the world, showing remarkable ecological diversity of habitats such as freshwater, terrestrial, marine, hot spring, etc. These BGA are also widely distributed in the polar region such as the Arctic, Antarctic, Southern Ocean, and the Himalayas. Several studies have been conducted for the isolation and identification of Blue-Green algae from the water, soil, sediments, algal mats, etc. using advanced morphological, physiological, and molecular characterization techniques. Various selective media are known for their Cultivation. It has been now proven that BGA offers a great opportunity as these are considered to be one of the potential organisms useful to mankind in many ways. They exhibit diverse biological activities (Antibacterial, Antifungal, Anticancer, Antiviral Antidiabetic, and many more). Various bioactive molecules have been reported by researchers globally. In pharmaceutical companies especially in the new drug discovery research division, for the last many year's research is going on at various levels starting from extraction, purification, and identification of new compounds or drugs from various species of BGA. The major challenge in front of the current world is to fight effectively against the new emerging diseases and microbes specifically WHO priorities list of multiple antibiotic-resistant bacteria, microbial infections including SARS-CoV-2 virus and Cancer, etc., and to discover new pharmaceutical compounds for mankind and society. At the same time, there is an urgent need to think from basic to applied research to commercialize several value-added products. The use of nanomaterials to enhance biological activity could be one of the ways. Inventions of these drugs using nanotechnology can lead to the development of novel pharmaceuticals. Based on the cultures of cells, activities of enzymes, and receptors binding with ligands, various new technologies are invented to develop novel things of miniaturized screens. As a result, there is a conformational analysis, i.e., an analysis of the spatial arrangement of the component atom within a molecule that can be rotated about one or more single bonds. The known ligands result in the development of new compounds of structure-based drug design. Hence, the pharmaceutical potential of blue-green algae deserves more scientific attention and interdisciplinary research, and BGA strains from still unexplored and extreme habitats such as the Antarctic, Arctic, and the Himalayas can serve as good candidates in this regard.

\section{Authors' contributions}

All authors contributed significantly to the conception and design of the study, the interpretation of data, and the drafting and revision of the manuscript. All authors read and approved the final manuscript.

\section{Acknowledgments}

The authors acknowledge the support of Dr. Ashok K. Chauhan, Founder President, Amity Universe, for his constant support and encouragement. The authors also thank Dr. Atul Chauhan, Chancellor, Amity University, Uttar Pradesh, for providing all the required facilities.

\section{Conflict of Interest}

The authors hereby declare no conflict of interest.

\section{Funding support}

The authors declare that they have no funding support for this study

\section{References}

Ahmed BE, Badawi MH, Mostafa SS, Higazy AM (2018) Human anticancer and antidiabetic activities of the cyanobacterium Fischerella sp. BS1-EG isolated from River Nile. Egypt. International Journal Current Microbiology and Applied Science 7(1): 3473-3485.

Allen MB, Arnon DI (1955) Studies on nitrogen fixing blue green algae. I. growth and nitrogen fixation in Anabaena cylindrca. Plant Physiology 30: 366-372.

Al-Saman MA, Doleib NM, Ibrahim MR, Nasr MY, Tayel AA, Hamouda RA (2020) In vitro and in vivo hypolipidemic properties of the aqueous extract of Spirulina platensis, cultivated in colored flasks under artificial illumination. PeerJ 8: e10366.

Andrianasolo EH, Gross H, Goeger D, Musafija-Girt M, McPhail K, Leal RM, Mooberry SL, Gerwick WH (2005) Isolation of swinholide A and related glycosylated derivatives from two field collections of marine cyanobacteria. Organic letters 7(7):1375-8.

Asthana RK, Tripathi MK, Srivastava A, Singh AP, Singh SP, Nath G, Srivastava R, Srivastava BS (2009) Isolation and identification of a new antibacterial entity from the Antarctic cyanobacterium Nostoc CCC 537. Journal of Applied Phycology 21(1):81-8.

Atanasov AG, Zotchev SB, Dirsch VM, Supuran CT (2021) Natural products in drug discovery: advances and opportunities. Nature Reviews Drug Discovery 20(3):200-216. doi: 10.1038/s41573-020-00114-z.

Ayehunie S, Belay A, Baba TW, Ruprecht RM (1998) Inhibition of HIV-1 replication by an aqueous extract of Spirulina platensis (Arthrospira platensis). Journal of Acquired Immune Deficiency Syndrome 18(1): 7-12.

Back S, Liang J (2005) Production of cryptophycin from bluegreen algae. Journal of Young Investigator 12:1654-1673. 
Banker R, Carmeli S (1998) Tenuecyclamides A- D, Cyclic Hexapeptides from the Cyanobacterium Nostoc spongiaeforme var. t enue. Journal of Natural Products 61(10):1248-51.

Basu S, Gledhill M, de Beer D, Matondkar SP, Shaked Y (2019) Colonies of marine cyanobacteria Trichodesmium interact with associated bacteria to acquire iron from dust. Communications Biology 2(1): 1-8.

Becher PG, Keller S, Jung G, Süssmuth RD, Jüttner F (2007) Insecticidal activity of 12-epi-hapalindole $\mathrm{J}$ isonitrile. Phytochemistry 68(19):2493-7.

Bellahcen TO, AAmiri A, Touam I, Hmimid F, El Amrani A, Cherif A, Cherki M (2020) Evaluation of Moroccan microalgae: Spirulina platensis as a potential source of natural antioxidants. Journal of Complementary and Integrative Medicine 17(3). https://doi.org/10.1515/jcim-2019-0036.

Bellinger EG, Sigee DC (2015) Freshwater algae: identification, enumeration and use as bioindicators. John Wiley \& Sons.

Bernardini S, Tiezzi A, LaghezzaMasci V, Ovidi E (2018) Natural products for human health: an historical overview of the drug discovery approaches. Natural Product Research 32(16): 19261950.

Berry JP, Gantar M, Gawley RE, Wang M, Rein KS (2004) Pharmacology and toxicology of pahayokolide A, a bioactive metabolite from a freshwater species of Lyngbya isolated from the Florida Everglades. Comparative Biochemistry and Physiology Part C: Toxicology \& Pharmacology 139(4):231-8.

Bewley CA (2001) Solution structure of a cyanovirin-N: Mana12Man $\alpha$ complex: structural basis for high-affinity carbohydratemediated binding to gp120. Structure 9(10):931-40.

Bhadury P, Wright PC (2004) Exploitation of marine algae: biogenic compounds for potential antifouling applications. Planta 219(4):561-78.

Bokesch HR, O'Keefe BR, McKee TC, Pannell LK, Patterson GM, Gardella RS, Sowder RC, Turpin J, Watson K, Buckheit RW, Boyd MR (2003) A potent novel anti-HIV protein from the cultured cyanobacterium Scytonemavarium. Biochemistry 42(9):2578-84.

Bold HC (1949) The morphology of Chlamydomonas chlamydogama, sp. nov. Bulletin of the Torrey Botanical Club $1: 101-8$

Bonjouklian R, Moore RE, Patterson GM (1988) Acid-catalyzed reactions of hapalindoles. The Journal of Organic Chemistry 53(25):5866-70.
Boyd MR, Gustafson K, McMahon JB, Shoemaker R (1996) Discovery of cyanovirin-N, a novel HIV-inactivating protein from Nostoc ellipsosporum that targets viral gp120. International Conference on AIDS 11: 71-71.

Boyd MR, Gustafson KR, McMahon JB, Shoemaker RH, O'Keefe BR, Mori T, Gulakowski RJ, Wu L, Rivera MI, Laurencot CM, Currens MJ (1997) Discovery of cyanovirin-N, a novel human immunodeficiency virus-inactivating protein that binds viral surface envelope glycoprotein gp120: potential applications to microbicide development. Antimicrobial Agents and Chemotherapy 41(7):1521-30.

Bui HT, Jansen R, Pham HT, Mundt S (2007) Carbamidocyclophanes A- E, Chlorinated Paracyclophanes with Cytotoxic and Antibiotic Activity from the Vietnamese Cyanobacterium Nostoc sp. Journal of Natural Products 70(4):499503.

Cardellina II JH, Kirkup MP, Moore RE, Mynderse JS, Seff K, Simmons CJ (1979a) Hyellazone and chlorohyellazole, two novel carbazoles from the blue-green alga hyellacaespitosa born. et flah. Tetrahedron Letters 20(51):4915-4916.

Cardellina JH, Marner FJ, Moore RE (1979b) Seaweed dermatitis: structure of lyngbyatoxin A. Science 204(4389):193-5.

Carmichael WW (1992) Cyanobacteria secondary metabolitesthe cyanotoxins. Journal of Applied Bacteriology 72(6):445-59.

Carpine R, Sieber S (2021) Antibacterial and antiviral metabolites from cyanobacteria: their application and their impact on human health. Current Research in Biotechnology 3: 65-81.

Chatterjee PM, Tiwari DP, Datta S, Chakrabarty S, Raval R, Dubey AK (2019) Probing into methylene blue interaction with polyglutamic acid: Spectroscopic and molecular dynamics simulation studies. Asian Journal of Chemistry 31(9): 1949-1958.

Chauhan A, Chauhan G, Gupta PC, Goyal P, Kaushik P (2010) In vitro antibacterial evaluation of Anabaena sp. against several clinically significant microflora and HPTLC analysis of its active crude extracts. Indian Journal of Pharmacology 42(2): 105.

Chauhan A, Jindal T (2020) Microbiological Methods for Water, Soil and Air Analysis. In: Microbiological Methods for Environment, Food and Pharmaceutical Analysis 2020, Springer, Cham, Pp. 93-196.

Chauhan A, Ranjan A, Basniwal RK, Jindal T (2022) Cytotoxic and Antibiotic Properties of Cyanobacterial Extracts. In: Jindal T (eds) New Frontiers in Environmental Toxicology. Springer, Cham. https://doi.org/10.1007/978-3-030-72173-2_4.

Journal of Experimental Biology and Agricultural Sciences http://www.jebas.org 
Chen X, Smith GD, Waring P (2003) Human cancer cell (Jurkat) killing by the cyanobacterial metabolite calothrixin A. Journal of Applied Phycology 15(4):269-77.

Cheng YI, Chou L, Chiu YF, Hsueh HT, Kuo CH, Chu HA (2020) Comparative genomic analysis of a novel strain of Taiwan hotspring cyanobacterium Thermosynechococcus sp. CL-1. Frontiers in microbiology 11: 82 .

Chittapun S, Jonjaroen V, Khumrangsee K, Charoenrat T (2020) C-phycocyanin extraction from two freshwater cyanobacteria by freeze thaw and pulsed electric field techniques to improve extraction efficiency and purity. Algal Research 46: 101789.

Choi H, Engene N, Smith JE, Preskitt LB, Gerwick WH (2010) Crossbyanols A- D, toxic brominated polyphenyl ethers from the Hawai'ian bloom-forming Cyanobacterium Leptolyngbyacross byana. Journal of Natural Products 73(4):517-22.

Chorus I, Falconer IR, Salas HJ, Bartram J (2000) Health risks caused by freshwater cyanobacteria in recreational waters. Journal of Toxicology and Environmental Health Part B: Critical Reviews 3(4):323-47.

Chu SP (1942) The influence of the mineral composition of the medium on the growth of planktonic algae: part I. Methods and culture media. The Journal of Ecology 1:284-325.

Dimri AG, Prasad R, Chauhan A, Aggarwal ML, Varma A (2018) Characterization of soil actinomycete isolate against gram-positive and gram-negative food borne bacteria. Indian Journal of Environmental Protection 38: 1004-1015.

Doan NT, Rickards RW, Rothschild JM, Smith GD (2000) Allelopathic actions of the alkaloid 12-epi-hapalindole $\mathrm{E}$ isonitrile and calothrixin A from cyanobacteria of the genera Fischerella and Calothrix. Journal of Applied Phycology 12(3):409-16.

Duy TN, Lam PKS, Shaw GR, Connell DW (2000) Toxicology and Risk Assessment of Freshwater Cyanobacterial (Blue-Green Algal) Toxins in Water. In: Ware GW (Eds.) Reviews of Environmental Contamination and Toxicology. Reviews of Environmental Contamination and Toxicology, vol 163. Springer, New York, NY. https://doi.org/10.1007/978-1-4757-6429-1_3.

El-Sheekh MM, Hassan LH, Morsi HH (2021) Evaluation of antimicrobial activities of blue-green algae-mediated silver and gold nanoparticles. RendicontiLincei. ScienzeFisiche e Naturali 18:1-3.

Falch BS, König GM, Wright AD, Sticher O, Angerhofer CK, Pezzuto JM, Bachmann H (1995) Biological activities of cyanobacteria: evaluation of extracts and pure compounds. Planta Medica 61(04):321-8.

Fatima N, Ahmad IZ, Chaudhry H (2017) Alterations in the antibacterial potential of Synechococcus spp. PCC7942 under the influence of UV-B radiations on skin pathogens. Saudi Journal of Biological Sciences 24(7): 1657-1662.

Fogg G (1965) Algal culture and phytoplankton ecology. University of Wisconsin Press, Madison Pp. 126.

Gabr GA, El-Sayed SM, Hikal MS (2020) Antioxidant Activities of Phycocyanin: A Bioactive Compound from Spirulina platensis. Journal of Pharmaceutical Research International 32(2): 73-85.

Gademann K, Portmann C (2008) Secondary metabolites from cyanobacteria: complex structures and powerful bioactivities. Current Organic Chemistry 12(4):326-341.

Gamal S, Abd El Baky HH, Saleh MA (2020) Egyptian Arthrospira phytosterols: production, identification, antioxidant and antiproliferative activities. Notulae Botanicae HortiAgrobotanici Cluj-Napoca 48(2): 666-680.

Gara-Ali M, Zili F, Hosni K, Ouada HB, Ben-Mahrez K (2021) Lipophilic extracts of the thermophilic cyanobacterium Leptolyngbya sp. and chlorophyte Graesiella sp. and their potential use as food and anticancer agents. Algal Research 60: 102511.

Gerber P, Dutcher JD, Adams EV, Sherman JH (1958) Protective effect of seaweed extracts for chicken embryos infected with influenza B or mumps virus. Proceedings of the Society for Experimental Biology and Medicine 99(3): 590-593.

Gerwick WH, Mrozek C, Moghaddam MF, Agarwal SK (1989) Novel cytotoxic peptides from the tropical marine cyanobacterium Hormothamnion enteromorphoides 1. Discovery, isolation and initial chemical and biological characterization of the hormothamnins from wild and cultured material. Experientia 45(2):115-21.

Gheda SF, Ismail GA (2020) Natural products from some soil cyanobacterial extracts with potent antimicrobial, antioxidant and cytotoxic activities. Anais da Academia Brasileira de Ciências 92(2):e20190934. doi: 10.1590/0001-3765202020190934.

Ghosh T, Bhayani K, Paliwal C, Maurya R, Chokshi K, Pancha I, Mishra S (2016) Cyanobacterial pigments as natural antihyperglycemic agents: an in vitro study. Frontiers in Marine Science 10(3): 146 .

Grinberg M, Sarig R, Zaltsman Y, Frumkin D, Grammatikakis N, Reuveny E, Gross A (2002) tBID Homooligomerizes in the 
mitochondrial membrane to induce apoptosis. Journal of Biological Chemistry 277(14):12237-45.

Guerreiro A, Andrade MA, Menezes C, Vilarinho F, Dias E (2020) Antioxidant and Cytoprotective Properties of Cyanobacteria: Potential for Biotechnological Applications. Toxins 12(9): 548.

Gunasekera SP, Ross C, Paul VJ, Matthew S, Luesch H (2008) Dragonamides $\mathrm{C}$ and $\mathrm{D}$, linear lipopeptides from the marine cyanobacterium brown Lyngbya polychroa. Journal of Natural products 71(5):887-90.

Gustafson KR, Cardellina II JH, Fuller RW, Weislow OS, Kiser RF, Sander KM, Patterson GML, Boyd MR (1989) AIDS-antiviral sulfolipids from cyanobacteria (blue-green algae). Journal of the National Cancer Institute 81: 1254-1258.

Gustafson KR, Sowder R, Henderson L, Pannel L, Cardellina J, McMahon J, Shoemaker R, Boyd M (1996) Sequence determination and chemical chractristics of a novel anti-HIV protein, Cyanovirin-N, isolated from the cyanobacterium Nostoc ellipsosporum. International Controversies on AIDS 11: 70.

Hayashi K, Hayashi T, Kojima I (1996) A natural sulfated polysaccharide, calcium spirulan, isolated from Spirulina platensis: in vitro and ex vivo evaluation of anti-herpes simplex virus and anti-human immunodeficiency virus activities. AIDS Research and Human Retroviruses 12(15):1463-71.

Hirata K, Yoshitomi S, Dwi S, Iwabe O, Mahakhant A, Polchai J, Miyamoto K (2003) Bioactivities of nostocine A produced by a freshwater cyanobacterium Nostoc spongiaeforme TISTR 8169. Journal of Bioscience and Bioengineering 95(5):512-7.

Hokmollahi F, Riahi H, Soltani N, Shariatmadari Z, Hakimi Meybodi MH (2016) A taxonomic study of blue-green algae based on morphological, physiological and molecular characterization in Yazd province terrestrial ecosystems (Iran). Rostaniha 16(2): 152163.

Hwang P, Hung Y, Tsai Y, Chien SY, Kong Z (2014) The brown seaweed Sargassum hemiphyllum exhibits $\alpha$-amylase and $\alpha$ glucosidase inhibitory activity and enhances insulin release in vitro. Cytotechnology 67: 653-660.

Inderjit DK, Dakshini KM (1994) Algal allelopathy. The Botanical Review 60:182-96.

Ishibashi M, Moore RE, Patterson GM, Xu C, Clardy J (1986) Scytophycins, cytotoxic and antimycotic agents from the cyanophyte Scytonemapseudohofmanni. The Journal of Organic Chemistry 51(26):5300-6.
Ishida K, Matsuda H, Murakami M, Yamaguchi K (1997) Kawaguchipeptin B, an antibacterial cyclic undecapeptide from the cyanobacterium Microcystis aeruginosa. Journal of Natural Products 60(7):724-6.

Jafari Porzani S, Konur O, Nowruzi B (2021) Cyanobacterial natural products as sources for antiviral drug discovery against COVID-19. Journal of Biomolecular Structure and Dynamics 1-17. doi: 10.1080/07391102.2021.1899050.

Jaki B, Orjala J, Heilmann J, Linden A, Vogler B, Sticher O (2000) Novel Extracellular Diterpenoids with Biological Activity from the Cyanobacterium Nostoc commune. Journal of natural products 63(3):339-43.

Jaki B, Zerbe O, Heilmann J, Sticher O (2001) Two novel cyclic peptides with antifungal activity from the cyanobacterium Tolypothrix byssoidea (EAWAG 195). Journal of Natural Products 64(2):154-8.

Jaspars M, Lawton LA (1998) Cyanobacteria-a novel source of pharmaceuticals. Current opinion in Drug Discovery \& Development (1):77-84.

Jimenez-Lopez C, Pereira AG, Lourenço-Lopes C, Garcia-Oliveira P, Cassani L, Fraga-Corral M, Simal-Gandara J (2021) Main bioactive phenolic compounds in marine algae and their mechanisms of action supporting potential health benefits. Food Chemistry 341: 128262.

Jo SW, Do JM, Na H, Hong JW, Kim IS, Yoon HS (2020) Assessment of biomass potentials of microalgal communities in open pond raceways using mass cultivation. PeerJ 8: e9418.

Jones GJ, Korth W (1995) In situ production of volatile odour compounds by river and reservoir phytoplankton populations in Australia. Water Science and Technology 31(11):145-51.

Kaiwan-arporn P, Hai PD, Thu NT, Annachhatre AP (2012) Cultivation of cyanobacteria for extraction of lipids. Biomass and Bioenergy 44: 142-149.

Kajiyama SI, Kanzaki H, Kawazu K, Kobayashi A (1998) Nostofungicidine, an antifungal lipopeptide from the field-grown terrestrial blue-green alga Nostoc commune. Tetrahedron Letters 39(22):3737-3740.

Kaushik P, Abhishek C, Pankaj G (2009) Screening of Lyngbyama juscula for potential antibacterial activity and HPTLC analysis of active methanolic extract. Journal of Pure and Applied Microbiology 3(1): 169-174. 
Kaushik P, Chauhan A (2008a) Antibacterial Potential of aqueous and organic extracts of N. Commune: Cyanobacterium. VEGETOS 21(1): 77-80.

Kaushik P, Chauhan A (2008b) In vitro antibacterial activity of laboratory grown culture of Spirulina platensis. Indian Journal of Microbiology 48(3): 348-352.

Kaushik P, Goyal P, Chauhan A, Chauhan G (2010) In vitro evaluation of antibacterial potential of dry fruit extracts of Elettaria cardamomum Maton (ChhotiElaichi). Iranian Journal of Pharmaceutical Research 9(3): 287.

Kehr JC, Zilliges Y, Springer A, Disney MD, Ratner DD, Bouchier C, Seeberger PH, De Marsac NT, Dittmann E (2006) A mannan binding lectin is involved in cell-cell attachment in a toxic strain of Microcystis aeruginosa. Molecular Microbiology 59(3):893906.

Khatoon H, Leong LK, Rahman NA, Mian S, Begum H, Banerjee S, Endut A (2018) Effects of different light source and media on growth and production of phycobiliprotein from freshwater cyanobacteria. Bioresource Technology 249: 652-658.

Larsen LK, Moore RE, Patterson GM (1994) $\beta$-Carbolines from the blue-green alga Dichothrix baueriana. Journal of Natural Products 57(3):419-21.

Lau AF, Siedlecki J, Anleitner J, Patterson GM, Caplan FR, Moore RE (1993) Inhibition of reverse transcriptase activity by extracts of cultured blue-green algae (cyanophyta). Planta Medica 59: 148151.

Li LY, Wu W, Chen S, Gu JW, Li XL, Song HJ, Du F, Wang G, Zhong CQ, Wang XY, Chen Y, Shah R, Yang HM, Cai Q (2020) Digestive system involvement of novel coronavirus infection: Prevention and control infection from a gastroenterology perspective. Journal of Digestive Diseases 21(4):199204.https://doi.org/10.1111/1751-2980.12862

Liu X, Lagenaur LA, Simpson DA, Essenmacher KP, FrazierParker CL, Liu Y, Tsai D, Rao SS, Hamer DH, Parks TP, Lee PP (2006) Engineered vaginal lactobacillus strain for mucosal delivery of the human immunodeficiency virus inhibitor cyanovirin-N. Antimicrobial Agents and Chemotherapy 50(10):3250-3259.

Loya S, Reshef V, Mizrrachi E, Silberstein C, Rachamim Y, Carmcli S, Hizi A (1998) The inhibition of the reverse transcriptase of HIV-1 by the natural sulfoglycolipids from cyanobacteria: contribution of different moieties to their high potency. Journal of Natural Product 61: 891-895.
Luesch H, Moore RE, Paul VJ, Mooberry SL, Corbett TH (2001) Isolation of dolastatin 10 from the marine cyanobacterium Symploca species VP642 and total stereochemistry and biological evaluation of its analogue symplostatin 1. Journal of Natural Products 64(7):907-10.

MacMillan JB, Ernst-Russell MA, De Ropp JS, Molinski TF (2002) Lobocyclamides A- C, Lipopeptides from a cryptic cyanobacterial mat containing Lyngbya c onfervoides. The Journal of Organic Chemistry 67(23):8210-5.

MacMillan JB, Molinski TF (2005) Majusculoic acid, a brominated cyclopropyl fatty acid from a marine cyanobacterial mat assemblage. Journal of Natural Products 68(4):604-6.

Marrez DA, Sultan YY (2016) Antifungal activity of the cyanobacterium Microcystis aeruginosa against mycotoxigenic fungi. Journal of Applied Pharmaceutical Science 6(11): 191-198.

Mason CP, Edwards KR, Carlson RE, Pignatello J, Gleason FK, Wood JM (1982) Isolation of chlorine-containing antibiotic from the freshwater cyanobacterium Scytonemahofmanni. Science 215(4531):400-2.

Matern U, Oberer L, Falchetto RA, Erhard M, König WA, Herdman M, Weckesser J (2001) Scyptolin A and B, cyclic depsipeptides from axenic cultures of Scytonema hofmanni PCC 7110. Phytochemistry 58(7):1087-95.

Mathew B, Sankaranarayanan R, Nair PP, Varghese C, Somanathan T, Amma BP, Amma NS, Nair MK(1995) Evaluation of chemoprevention of oral cancer with Spirulina fusiformis. Nutrition and Cancer 24: 197-202

Medina RA, Goeger DE, Hills P, Mooberry SL, Huang N, Romero LI, Ortega-Barría E, Gerwick WH, McPhail KL (2008) Coibamide A, a potent antiproliferative cyclic depsipeptide from the Panamanian marine cyanobacterium Leptolyngbya sp. Journal of the American Chemical Society 130(20):6324-5.

Metting B, Pyne JW (1986) Biologically active compounds from microalgae. Enzyme and Microbial Technology 8(7):386-94.

Mishima T, Murata J, Toyoshima M, Fujii H, Nakajima M, Hayashi T, Kato T, Saiki I (1998) Inhibition of tumor invasion and metastasis by calciumspirulan (Ca-SP), a novel sulfated polysaccharide derived from a blue-green alga, Spirulina platensis. Clinical \& Experimental Metastasis 16(6):541-550.

Mo S, Krunic A, Chlipala G, Orjala J (2009) Antimicrobial ambiguine isonitriles from the cyanobacterium Fischerella ambigua. Journal of Natural Products 72(5):894-9. 
Moon SS, Chen JL, Moore RE, Patterson GM (1992) Calophycin, a fungicidal cyclic decapeptide from the terrestrial blue-green alga Calothrix fusca. The Journal of Organic Chemistry 57(4):1097103.

Moore RE (1982) Toxins, anticancer agents, and tumor promoters from marine prokaryotes. Pure and Applied Chemistry 54(10):1919-34.

Moore RE, Patterson GM, Carmichael WW (1988) New pharmaceuticals from cultured blue-green algae. Biomedical Importance of Marine Organisms 13:143-50.

Moore RE, Yang XQ, Patterson GM (1987) Fontonamide and anhydrohapaloxindole A, two new alkaloids from the blue-green alga Hapalosiphon fontinalis. The Journal of Organic Chemistry 52(17):3773-3777.

Müller D, Krick A, Kehraus S, Mehner C, Hart M, Küpper FC, Saxena K, Prinz H, Schwalbe H, Janning P, Waldmann H (2006) Brunsvicamides A- C: Sponge-Related Cyanobacterial Peptides with Mycobacterium tuberculosis Protein Tyrosine Phosphatase Inhibitory Activity. Journal of Medicinal Chemistry 49(16):48718.

Mundt S, Kreitlow S, Jansen R (2003) Fatty acids with antibacterial activity from the cyanobacterium Oscillatoria redeke HUB 051. Journal of Applied Phycology 15(2):263-7.

Nagatsu A, Kajitani H, Sakakibara J. Muscoride A (1995) A new oxazole peptide alkaloid from freshwater cyanobacterium Nostoc muscorum. Tetrahedron Letters 36(23):4097-100.

Nainangu P, Antonyraj APM, Subramanian K, Kaliyaperumal S, Gopal S, Renuka PS (2020) In vitro screening of antimicrobial, antioxidant, cytotoxic activities, and characterization of bioactive substances from freshwater cyanobacteria Oscillatoria sp. SSCM01 and Phormidium sp. SSCM02. Biocatalysis and Agricultural Biotechnology 29: 101772.

Natarajan C, Prasanna R, Gupta V, Dureja P, Nain L (2012) Characterization of the fungicidal activity of Calothrix elenkinii using chemical methods and microscopy. Applied Biochemistry and Microbiology 48(1):51-57.

Neuhof T, Schmieder P, Preussel K, Dieckmann R, Pham H, Bartl F, von Döhren H (2005) Hassallidin A, a glycosylated lipopeptide with antifungal activity from the cyanobacterium Hassallia $s p$ Journal of Natural Products 68(5):695-700.

Niveshika, Verma E, Mishra AK, Singh AK, Singh VK (2016) Structural elucidation and molecular docking of a novel antibiotic compound from cyanobacterium Nostoc sp. MGL001. Frontiers in microbiology 7:1899.

Padmini N, Ajilda AA, Prabakaran P, Sivakumar N, Selvakumar G (2021) Efficacy of marine cyanobacterium Oxynemathaianum ALU PBC5 against multi drug resistant Gram negative pathogens. Journal of Applied Microbiology 130(1):50-60.

Petersen LE, Kellermann MY, Schupp PJ (2020) Secondary metabolites of marine microbes: From natural products chemistry to chemical ecology. Youmares 9-The Oceans: Our Research, Our Future 159.

Ploutno A, Carmeli S (2000) Nostocyclyne A, a novel antimicrobial cyclophane from the cyanobacterium Nostoc sp. Journal of Natural Products 63(11):1524-6.

Pratt R, Daniels TC, Eiler JJ, Gunnison JB, Kumler WD, Oneto JF, Strait LA, Spoehr HA, Hardin GJ, Milner HW, Smith JH (1944) Chlorellin, an antibacterial substance from Chlorella. Science 99 (2574):351-352.

Priatni S, Budiwati TA, Ratnaningrum D, Kosasih W, Andryani R, Susanti H, Susilaningsih W (2016) Antidiabetic screening of some Indonesian marine cyanobacteria collection. Biodiversitas 17(2): 641-646.

Pringsheim E (1946) Pure Cultures of Algae. Cambridge University Press London Pp. 119.

Prinsep MR, Moore RE, Levine IA, Patterson GM (1992) Westiellamide, a bistratamide-related cyclic peptide from the bluegreen alga Westiellopsis prolifica. Journal of Natural Products 55(1):140-2.

Radkova M, Stefanova K, Uzunov B, Gärtner G, Stoyneva-Gärtner M (2020) Morphological and molecular identification of microcystin-producing cyanobacteria in nine shallow Bulgarian water bodies. Toxins 12(1): 39 .

Radzi R, Muangmai N, Broady P, Wan Omar WM, Lavoue S, Convey P, Merican F (2019) Nodosilineasigniensis sp. Nov. (Leptolyngbyaceae, Synechococcales), a new terrestrial cyanobacterium isolated from mats collected on Signy Island, South Orkney Islands, Antarctica. PloSone 14(11): e0224395.

Raveh A, Carmeli S (2007) Antimicrobial ambiguines from the cyanobacterium Fischerella sp. collected in Israel. Journal of Natural Products 70(2):196-201.

Rego A, Raio F, Martins TP, Ribeiro H, Sousa AG, Séneca J, Magalhães C (2019) Actinobacteria and cyanobacteria diversity in terrestrial antarctic microenvironments evaluated by culture- 
dependent and independent methods. Frontiers in Microbiology 10: 1018.

Reshef V, Mizrachi E, Maretzki T, Silberstein C, Loya S, Hizi A, Carmeli S (1997) New acetylated sulfoglycolipids and digalactolipids and related known glycolipids from cyanobacteria with potential to inhibit the reverse transcriptase of HIV-1. Journal of Natural Products 60: 1251-1260.

Riba M, Kiss-Szikszai A, Gonda S, Parizsa P, Deák B, Török P, Vasas G (2020) Chemotyping of terrestrial Nostoc-like isolates from alkali grassland areas by non-targeted peptide analysis. Algal Research 46: 101798.

Rippka R, Deruelles J, Waterbury JB, Herdman M, Stanier RY (1979) Generic assignments, strain histories and properties of pure cultures of cyanobacteria. Microbiology 111: 1- 61.

Romay C, Ledon N, Genzalez R (1999) Phycocyanin extract reduces leukotriene B4 levels in arachidonic acid-induced mouseearinflammation test. Journal of Pharmacy and Pharmacology 51(5): 641-642.

Safari R, Raftani Amiri Z, Esmaeilzadeh Kenari R (2020) Antioxidant and antibacterial activities of C-phycocyanin from common name Spirulina platensis. Iranian Journal of Fisheries Sciences 19(4): 1911-1927.

Sami N, Ahmad R, Fatma T (2021) Exploring algae and cyanobacteria as a promising natural source of antiviral drug against SARS-CoV-2 Biomedical Journal 44(1) 54-62.

Saurav K, Macho M, Kust A, Delawská K, Hájek J, Hrouzek P (2019) Antimicrobial activity and bioactive profiling of heterocytous cyanobacterial strains using MS/MS-based molecular networking. Folia Microbiologica 64(5): 645-654.

Schaeffer DJ, Krylov VS (2000) Anti-HIV activity of extracts and compounds from algae and cyanobacteria. Ecotoxicology and Environmental safety 45(3):208-27.

Schwartz J, Shklar G, Reid S, and Trickler D (1988) Prevention of experimental oral cancer by extracts of Spirulina-Dunaliella algae. Nutrition and Cancer 11 (2); 127-134.

Seddek NH, Fawzy MA, El-Said WA, Ahmed MMR (2019) Evaluation of antimicrobial, antioxidant and cytotoxic activities and characterization of bioactive substances from freshwater bluegreen algae. Global Nest Journal 21(3): 328-336.

Senousy HH, Abd Ellatif S, Ali S (2020) Assessment of the antioxidant and anticancer potential of different isolated strains of cyanobacteria and microalgae from soil and agriculture drain water. Environmental Science and Pollution Research 27: 1846318474.

Shi SR, Cote RJ, Taylor CR (1999) Standardization and further development of antigen retrieval immunohistochemistry: strategies and future goals. Journal of Histotechnology 22(3):177-92.

Shishido TK, Popin RV, Jokela J, Wahlsten M, Fiore MF, Fewer DP, Herfindal L, Sivonen K (2020) Dereplication of natural products with antimicrobial and anticancer activity from Brazilian cyanobacteria. Toxins 12(1):12.DOI: 10.3390/toxins12010012.

Singh IP, Milligan KE, Gerwick WH (1999) Tanikolide, a toxic and antifungal lactone from the marine cyanobacterium Lyngbya m ajuscula. Journal of Natural Products 62(9):1333-5.

Singh SM, Elster JOSEF (2007) Cyanobacteria in Antarctic lake environments. In: Algae and cyanobacteria in extreme environments. Springer, Dordrecht Pp. 303-320.

Singh U, Singh AK, Asthana RK (2021a) Bioactive molecules from microalgae and constraints in commercialization. Natural Bioactive Compounds Pp. 143-164.

Singh U, Singh P, Singh AK, Kumar D, Tilak R, Shrivastava SK, Asthana RK (2021b) Identification of antifungal and antibacterial biomolecules from a cyanobacterium, Arthrospira platensis. Algal Research 54:102215.

Smetana S, Sandmann M, Rohn S, Pleissner D, Heinz V (2017) Autotrophic and heterotrophic microalgae and cyanobacteria cultivation for food and feed: life cycle assessment. Bioresource Technology 245: 162-170.

Spoehr HA, Milner HW (1949) The chemical composition of Chlorella; effect of environmental conditions. Plant physiology 24(1):120.

Sridhar K, Inbaraj BS, Chen BH(2021) Recent developments on production, purification and biological activity of marine peptides. Food Research International 147: p.110468.

Srivastava VC, Manderson GJ, Bhamidimarri R (1999) Inhibitory metabolites production by the cyanobacterium Fischerellamuscicola. Microbiological Research 153(4):309-17.

Stanier G (1988) Fine structure of cyanobacteria. Methods in Enzymology 167:157-172.

Stevenson CS, Capper EA, Roshak AK, Marquez B, Eichman C, Jackson JR, Mattern M, Gerwick WH, Jacobs RS, Marshall LA (2002) The identification and characterization of the marine natural product scytonemin as a novel antiproliferative pharmacophore. 
Journal of Pharmacology and Experimental Therapeutics 303(2):858-66.

Stratmann K, Moore RE, Bonjouklian R, Deeter JB, Patterson GM, Shaffer S, Smith CD, Smitka TA (1994) Welwitindolinones, unusual alkaloids from the blue-green algae Hapalosiphon welwitschii and Westiellaintricata. Relationship to fischerindoles and hapalinodoles. Journal of the American Chemical Society 116(22):9935-9942.

Sturdy M, Krunic A, Cho S, Franzblau S, Orjala J (2010) Eucapsitrione, an anti-Mycobacterium tuberculosis anthraquinone derivative from the cultured freshwater cyanobacterium Eucapsis $s p$. Journal of natural products 73(8):1441-3.

Tang J, Jiang D, Luo Y, Liang Y, Li L, Shah MMR, Daroch M (2018) Potential new genera of cyanobacterial strains isolated from thermal springs of western Sichuan, China. Algal Research 31: 1420 .

Thajuddin N, Subramanian G (2005) Cyanobacterial biodiversity and potential applications in biotechnology. Current Science 10:47-57.

Tokuda H, Nishino H, Shirahashi H, Murakami N, Nagatsu A, Sakakibara J (1996) Inhibition of 12-O-tetradecanooylphorbol-13acetate promoted mouse skin papilloma by digalactosyl diacylglycerols from the fresh water cyanobacterium Phormidium tenue. Cancer Letter 104: 91-95.

Trimurtulu G, Ohtani I, Patterson GM, Moore RE, Corbett TH, Valeriote FA, Demchik L (1994) Total structures of cryptophycins, potent antitumor depsipeptides from the blue-green alga Nostoc $\mathrm{sp}$ strain GSV 224. Journal of the American Chemical Society 116(11):4729-37.

Troschl C, Fritz I, Sodnikar K, Drosg B (2017) Contaminations in mass cultivation of cyanobacteria: Highly resilient Colpoda steinii leads to rapid crash of Synechocystis sp. cultures and is inhibited by partially anoxic conditions. Algal research 28: 229-234.

Uma VS, Gnanasekaran D, Lakshmanan U, Dharmar P (2020) Survey and isolation of marine cyanobacteria from eastern coast of India as a biodiesel feedstock. Biocatalysis and Agricultural Biotechnology 24: 101541.

Vanlalveni C, Rajkumari K, Biswas A, Adhikari PP, Lalfakzuala R, Rokhum L (2018) Green synthesis of silver nanoparticles using Nostoc linckia and its antimicrobial activity: a novel biological approach. BioNanoScience 8(2): 624-631.

Vasudevan S, Arulmoorthy MP, Suresh R (2020) Isolation, purification and structural elucidation of secondary metabolites from Microcystis aeruginosa bloom from Muttukadu estuary and its in vitro antibacterial, antioxidant and anticancer potency. South African Journal of Botany 132: 59-67.

Verma E, Mishra AK, Singh AK, Singh VK (2016) Structural elucidation and molecular docking of a novel antibiotic compound from cyanobacterium Nostoc sp. MGL001. Frontiers in Microbiology 29(7):1899.

Volk RB, Furkert FH (2006) Antialgal, antibacterial and antifungal activity of two metabolites produced and excreted by cyanobacteria during growth. Microbiological Research 161(2):180-6.

WHO (2017) Global priority list of Antibiotic resistant bacteria to guide research discovery and developments of new antibiotics.

Wright AD, Papendorf O, König GM (2005) Ambigol C and 2, 4Dichlorobenzoic Acid, Natural Products Produced by the Terrestrial Cyanobacterium Fischerella ambigua. Journal of Natural Products 68(3):459-461.

Xiong C, O'Keefe BR, Byrd RA, McMahon JB (2006) Potent antiHIV activity of scytovirin domain 1 peptide. Peptides 27(7):166875.

Xu HL, Kitajim C, Ito H, Miyazaki T, Bab M, Okuyam T (2012) Antidiabetic effect of polyphenols from brown alga Ecklonia kurome in genetically diabetic KK-Ay mice, Pharmaceutical Biology 50: 393-400.

Yamaguchi M, Ogawa T, Muramoto K, Kamio Y, Jimbo M, Kamiya (1999) Isolation and characterization of a mannan-binding lectin from the freshwater cyanobacterium (blue-green algae) Microcystis viridis. Biochemical and Biophysical Research Communications 265(3):703-8.

Yoon SH, Lee YM, Kim JE, Lee SH, Lee JH, Kim JY, Jung KH, Shin YC, Keasling JD, Kim SW (2006) Enhanced lycopene production in Escherichia coli engineered to synthesize isopentenyl diphosphate and dimethylallyl diphosphate from mevalonate. Biotechnology and Bioengineering 94(6):1025-32.

Yücer TD, Beyatlı Y, Pabuçcu K (2018) The antiproliferative and antimicrobial effects of cultivated Anabaena circinalis Rabenhorts ex Bornet and Flahault and Nostoc entophytum Bornet and Flahault. Tropical Journal of Pharmaceutical Research 17(8): 1571-1577.

Zaki S, Merican F, Muangmai N, Convey P, Broady P (2020). Discovery of microcystin-producing Anagnostidinema pseudacutissimum from cryopreserved Antarctic cyanobacterial mats. Harmful algae 93: 101800. 